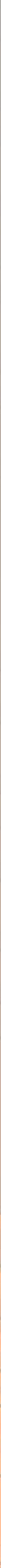




\title{
Conjugacy classes of special automorphisms of the affine spaces
}

\author{
Jérémy Blanc
}

In the group of polynomial automorphisms of the plane, the conjugacy class of an element is closed if and only if the element is diagonalisable. In this article, we show that this does not hold for the group of special automorphisms, giving a first step in the direction of showing that this group is not simple, as an infinite-dimensional algebraic group.

\section{Introduction}

In this article, $\mathrm{k}$ will always denote an algebraically closed field. The conjugacy classes of the algebraic groups $\operatorname{GL}(n, \mathrm{k})$ and $\operatorname{SL}(n, \mathrm{k})$ are well known. In particular, the following observation is classical:

An element is diagonalisable if and only if its conjugacy class is Zariski-closed.

As observed in [Furter and Maubach 2010], the same holds for the group $\operatorname{Aut}\left(\mathbb{A}_{\mathbb{C}}^{2}\right)$ of complex polynomial automorphisms of the affine plane. Here, the topology corresponds to the topology of $\operatorname{Aut}\left(\mathbb{A}_{\mathrm{k}}^{n}\right)$ induced by families parametrised by algebraic varieties $A$, called morphisms $A \rightarrow \operatorname{Aut}\left(\mathbb{A}_{\mathrm{k}}^{n}\right)$ and corresponding to elements of $\operatorname{Aut}\left(\mathbb{A}_{\mathrm{k}[A]}^{n}\right)$ (see Section $\left.2 \mathrm{~A}\right)$.

In fact, there is one easy direction in the result of [Furter and Maubach 2010], which corresponds to showing that if the conjugacy class is closed, then the element is diagonalisable. This works over any algebraically closed field $\mathrm{k}$ and follows from the following observation: If $f \in \operatorname{Aut}\left(\mathbb{A}_{\mathrm{k}}^{n}\right)$ is an element that fixes the origin, the conjugation of $f$ by

$$
\left(x_{1}, \ldots, x_{n}\right) \longmapsto\left(\frac{1}{t} x_{1}, \ldots, \frac{1}{t} x_{n}\right)
$$

The author gratefully acknowledges support by the Swiss National Science Foundation Grant "Birational Geometry" PP00P2_153026 /1 and by the French National Research Agency Grant "BirPol", ANR-11-JS01-004-01.

MSC2010: primary 14R10; secondary 14R20.

Keywords: plane automorphisms, conjugacy classes, topology. 
yields an element of $\operatorname{Aut}\left(\mathbb{A}_{\mathrm{k}(t)}^{n}\right)$ whose value at $t=0$ is the linear part of $f$, which is an element of $\operatorname{GL}(n, \mathrm{k})$. Moreover elements of $\operatorname{Aut}\left(\mathbb{A}_{\mathrm{k}}^{2}\right)$ which do not have fixed points are easy to handle (these are conjugate to $\left(x_{1}, x_{2}\right) \mapsto\left(x_{1}+1, a x_{2}+P\left(x_{2}\right)\right)$ for some polynomial $P \in \mathrm{k}\left[x_{2}\right]$ and $\left.a \in \mathrm{k}^{*}\right)$.

In this article, we focus on the closed normal subgroup $\operatorname{SAut}\left(\mathbb{A}_{\mathrm{k}}^{n}\right)$ of $\operatorname{Aut}\left(\mathbb{A}_{\mathrm{k}}^{n}\right)$ of elements of Jacobian 1. We will show that the conjugacy classes of the two groups $\operatorname{SAut}\left(\mathbb{A}_{\mathrm{k}}^{n}\right)$ and $\operatorname{Aut}\left(\mathbb{A}_{\mathrm{k}}^{n}\right)$ have a very different behaviour.

We say that an element $f \in \operatorname{Aut}\left(\mathbb{A}_{\mathrm{k}}^{n}\right)$ is dynamically regular if the extensions of $f$ and $f^{-1}$ to $\mathbb{P}_{\mathrm{k}}^{n}$ have disjoint indeterminacy loci (see Section $2 \mathrm{~B}$ ). We will also say that $f$ is algebraic if $\left\{\operatorname{deg}\left(f^{n}\right)\right\}_{n \in \mathbb{N}}$ is bounded. The dynamically regular elements are never algebraic, and in dimension 2, nonalgebraic elements are conjugate to dynamically regular elements (see Remark 4.3).

The first result that we obtain is to show that there is no degeneration of conjugates of dynamically regular elements in $\operatorname{SAut}\left(\mathbb{A}_{\mathrm{k}}^{n}\right)$, contrary to the case of $\operatorname{Aut}\left(\mathbb{A}_{\mathrm{k}}^{n}\right)$.

Theorem 1.1. Let $f \in \operatorname{SAut}\left(\mathbb{A}_{\mathrm{k}}^{n}\right)$ be a dynamically regular element.

(1) If $\alpha \in \operatorname{SAut}\left(\mathbb{A}_{\mathrm{k}((t))}^{n}\right)$ is such that $\alpha f \alpha^{-1}$ has a value at $t=0$, then this value is conjugate to $f$ by $\alpha(0)$ in $\operatorname{SAut}\left(\mathbb{A}_{\mathrm{k}}^{n}\right)$ (in particular $\alpha$ is defined at $t=0$ ).

(2) For each integer $d$, the set $\left\{g f g^{-1} \mid g \in \operatorname{SAut}\left(\mathbb{A}_{\mathrm{k}}^{n}\right), \operatorname{deg}(g) \leq d\right\}$ is closed.

(3) If $n=2$, the conjugacy class of $f$ in $\operatorname{SAut}\left(\mathbb{A}_{\mathrm{k}}^{n}\right)$ is closed.

(4) If $\mathrm{k}$ is uncountable, the following holds: for each morphism $A \rightarrow \operatorname{SAut}\left(\mathbb{A}_{\mathrm{k}}^{n}\right)$, where $A$ is an algebraic variety, the preimage of the conjugacy class of $f$ contains the closure of each locally closed subset $B \subset A$ that it contains.

Remark 1.2. The proof of this result is given in Section 3. As we will show, part (1) implies the others.

In the notation of [Furter and Kraft $\geq 2016$ ], assertion (4) can be reinterpreted by saying that the conjugacy class $C(f)$ of a dynamically regular element $f$ is weakly closed in $\operatorname{SAut}\left(\mathbb{A}_{\mathrm{k}}^{n}\right)$.

In dimension 2, an easy consequence of Theorem 1.1 and of the Jung-van der Kulk theorem is the fact the conjugacy class of nonalgebraic elements of $\operatorname{SAut}\left(\mathbb{A}_{\mathrm{k}}^{2}\right)$ is in fact closed, contrary to the case of $\operatorname{Aut}\left(\mathbb{A}_{\mathrm{k}}^{2}\right)$. In fact, we can be much more precise: we describe in Section 4 the conjugacy classes of elements in $\operatorname{SAut}\left(\mathbb{A}_{k}^{2}\right)$, and decide which ones are closed. In particular, we obtain the following complete description.

Theorem 1.3. Let $f \in \operatorname{SAut}\left(\mathbb{A}_{\mathrm{k}}^{2}\right)$. Then:

(1) If $f$ is diagonalisable, its conjugacy class is closed. 
(2) If $f$ is algebraic but not diagonalisable, its conjugacy class is not closed. More precisely, there exists an element $F \in \operatorname{SAut}\left(\mathbb{A}_{\mathrm{k}[t]}^{2}\right)$ such that for each $t \neq 0$, $F(t) \in \operatorname{SAut}\left(\mathbb{A}_{K}^{2}\right)$ is conjugate to $f$, and $F(0) \in \operatorname{SAut}\left(\mathbb{A}_{\mathrm{k}}^{2}\right)$ is diagonalisable.

(3) If $f$ is not algebraic, its conjugacy class is closed.

Remark 1.4. Since the set $\operatorname{SAut}\left(\mathbb{A}_{\mathrm{k}}^{2}\right)_{\text {alg }}$ of algebraic elements of $\operatorname{SAut}\left(\mathbb{A}_{\mathrm{k}}^{2}\right)$ is closed (Corollary 4.4), we can $\operatorname{decompose} \operatorname{SAut}\left(\mathrm{A}_{\mathrm{k}}^{2}\right)$ as an infinite union of disjoint closed sets, one being $\operatorname{SAut}\left(\mathbb{A}_{\mathrm{k}}^{2}\right)_{\text {alg }}$ and the others being conjugacy classes of nonalgebraic elements. The group $\operatorname{SAut}\left(\mathbb{A}_{\mathrm{k}}^{2}\right)$ is however irreducible, by the simple observation made above.

Remark 1.5. Note that these results show that the group $\operatorname{SAut}\left(\mathbb{A}_{\mathrm{k}}^{2}\right)$ is more rigid than the group $\operatorname{Aut}\left(\mathbb{A}_{\mathrm{k}}^{2}\right)$, in the sense that there are less possible degenerations of conjugates.

One can check, using the conjugations around fixed points as above, that every normal closed subgroup of $\operatorname{Aut}\left(\mathbb{A}_{\mathrm{k}}^{2}\right)$ is either trivial, $\operatorname{SAut}\left(\mathbb{A}_{\mathrm{k}}^{2}\right)$ or $\operatorname{Aut}\left(\mathbb{A}_{\mathrm{k}}^{2}\right)$. The interesting question is then to know whether $\operatorname{SAut}\left(\mathbb{A}_{\mathrm{k}}^{2}\right)$ contains nontrivial closed normal subgroups (by [Furter and Lamy 2010], it contains many nontrivial normal subgroups which contain only nonalgebraic elements, and the identity).

The fact that the conjugacy classes of nonalgebraic elements are closed suggests that $\operatorname{SAut}\left(\mathbb{A}_{\mathrm{k}}^{2}\right)$ could contain nontrivial closed normal subgroups. This text can then be viewed as a first step towards the study of the simplicity of $\operatorname{SAut}\left(\mathbb{A}_{\mathrm{k}}^{n}\right)$, viewed as an infinite-dimensional algebraic group (ind-group). In [Shafarevich 1966], it is claimed that this one is simple, but the proof contains serious gaps.

\section{Preliminaries}

As we said, in the sequel $\mathrm{k}$ will always be an algebraically closed field. We will sometimes also work on a general field (most of the time with an extension of $\mathrm{k}$ ), and will denote it by $K$.

In this section, we introduce the terminology and give some basic results (most of them classical, maybe in alternate formulations) on the topology of $\operatorname{Aut}\left(\mathbb{A}_{\mathrm{k}}^{n}\right)$ (Section 2A), the relation between the iterations of a map and the indeterminacy sets at infinity (Section 2B) and the families of automorphisms parametrised by formal series (Section 2C), that we will need in Sections 3 and 4.

\section{A. Topology on $\operatorname{Aut}\left(\mathbb{A}_{k}^{n}\right)$.}

Notation 2.1. Let $R$ be any commutative unitary ring.

(1) We denote by $\operatorname{End}\left(\mathbb{A}_{R}^{n}\right)$ the set of algebraic endomorphisms of $\mathbb{A}_{R}^{n}$. An element $f \in \operatorname{End}\left(\mathbb{A}_{R}^{n}\right)$ is given by

$$
f:\left(x_{1}, \ldots, x_{n}\right) \longmapsto\left(f_{1}\left(x_{1}, \ldots, x_{n}\right), \ldots, f_{n}\left(x_{1}, \ldots, x_{n}\right)\right)
$$


for some polynomials $f_{1}, \ldots, f_{n} \in R\left[x_{1}, \ldots, x_{n}\right]$. The degree of $f$ is by definition the maximal degree of the $f_{i}$, and we will use the notation

$$
f=\left(f_{1}, \ldots, f_{n}\right) .
$$

This corresponds to a natural bijection $\operatorname{End}\left(\mathbb{A}_{R}^{n}\right) \rightarrow\left(R\left[x_{1}, \ldots, x_{n}\right]\right)^{n}$.

(2) The group $\operatorname{Aut}\left(\mathbb{A}_{R}^{n}\right)$ is equal to the group of automorphisms of $\mathbb{A}_{R}^{n}$, i.e., to the elements of $\operatorname{End}\left(\mathbb{A}_{R}^{n}\right)$ that admit an inverse in this set.

(3) For each $f \in \operatorname{End}\left(\mathbb{A}_{R}^{n}\right)$, we denote by

$$
\operatorname{Jac}(f)=\operatorname{det}\left(\frac{\partial f_{i}}{x_{j}}\right)_{i, j=1}^{n} \in R\left[x_{1}, \ldots, x_{n}\right]
$$

the Jacobian of $f$, and denote by $\operatorname{SAut}\left(\mathbb{A}_{R}^{n}\right)$, the normal subgroup of $\operatorname{Aut}\left(\mathbb{A}_{R}^{n}\right)$ given by $\left\{f \in \operatorname{Aut}\left(\mathbb{A}_{R}^{n}\right) \mid \operatorname{Jac}(f)=1\right\}$.

(4) We denote by $\operatorname{End}\left(\mathbb{A}_{R}^{n}\right)_{\leq d}$ and $\operatorname{Aut}\left(\mathbb{A}_{R}^{n}\right)_{\leq d}$ the subsets of $\operatorname{End}\left(\mathbb{A}_{R}^{n}\right)$ and $\operatorname{Aut}\left(\mathbb{A}_{R}^{n}\right)$ respectively, given by elements of degree $\leq d$.

Example 2.2. For each $p_{1} \in R\left[x_{1}\right], p_{2} \in R\left[x_{1}, x_{2}\right], \ldots, p_{n-1} \in R\left[x_{1}, \ldots, x_{n-1}\right]$ and $a_{1}, \ldots, a_{n} \in R^{*}$ the element

$$
\left(a_{1} x_{1}, a_{2} x_{2}+p_{1}, a_{3} x_{3}+p_{2}, \ldots, a_{n} x_{n}+p_{n-1}\right)
$$

belongs to $\operatorname{Aut}\left(\mathbb{A}_{R}^{n}\right)$. Such elements are usually called triangular, or de Jonquières.

Remark 2.3. Suppose that $R$ is a field $K$. Extending the scalars to an algebraically closed field, we observe that the Jacobian matrix of every element of $\operatorname{Aut}\left(\mathbb{A}_{K}^{n}\right)$ is invertible everywhere, $\operatorname{so} \operatorname{Jac}(f) \in K^{*}$. In particular,

$$
\operatorname{Aut}\left(\mathbb{A}_{K}^{n}\right) \subset\left\{f \in \operatorname{End}\left(\mathbb{A}_{K}^{n}\right) \mid \operatorname{Jac}(f) \in K^{*}\right\},
$$

and the equality, when $K$ is of characteristic zero, is the classical Jacobian conjecture, open for any $n \geq 2$.

If $Z$ is an algebraic variety defined over $\mathrm{k}$, where $\mathrm{k}$ is algebraically closed as before, there is a natural way to endow the group $\operatorname{Bir}(Z)$ of birational transformations of $Z$ with a topology (see for example [Demazure 1970; Serre 2010; Blanc 2010; Blanc and Furter 2013]). When restricted to the subgroup $\operatorname{Aut}(Z)$ of automorphisms, we obtain the following:

Definition 2.4. Let $A, Z$ be two algebraic varieties defined over k. We say that a morphism $f: A \rightarrow \operatorname{Aut}(Z)$ is a map given by an $A$-automorphism of $A \times Z$.

The Zariski topology on $\operatorname{Aut}(Z)$ is defined as follows: a set $F \subset \operatorname{Aut}(Z)$ is closed if and only if $f^{-1}(F) \subset A$ is closed for any algebraic variety $A$ and any morphism $f: A \rightarrow \operatorname{Aut}(Z)$. 
Remark 2.5. When the group $\operatorname{Aut}(Z)$ has a natural structure of an algebraic group (for example when $Z=\mathbb{P}^{n}$ ), the topology defined above agrees with the classical topology of the algebraic group, and morphisms $A \rightarrow \operatorname{Aut}(Z)$ correspond to morphisms of algebraic varieties.

However, in general the group $\operatorname{Aut}(Z)$ is too big to be an algebraic variety, for instance for $Z=\mathbb{A}^{n}, n \geq 2$ (see Example 2.2).

We will observe (in Lemma 2.7 below) that this topology, when restricted to $\operatorname{Aut}\left(\mathbb{A}_{\mathrm{k}}^{n}\right)$, gives the one introduced by Shafarevich in [Shafarevich 1966], i.e., the inductive limit topology given by the inclusion of affine algebraic varieties

$$
\operatorname{Aff}\left(\mathbb{A}_{\mathrm{k}}^{n}\right)=\operatorname{Aut}\left(\mathbb{A}_{\mathrm{k}}^{n}\right)_{\leq 1} \subset \operatorname{Aut}\left(\mathbb{A}_{\mathrm{k}}^{n}\right)_{\leq 2} \subset \operatorname{Aut}\left(\mathbb{A}_{\mathrm{k}}^{n}\right)_{\leq 3} \subset \cdots
$$

and corresponds in fact to an infinite-dimensional algebraic group. In order to do this, we recall how one obtains natural structures of affine varieties for $\operatorname{Aut}\left(\mathbb{A}_{\mathrm{k}}^{n}\right)_{\leq d}$ and $\operatorname{SAut}\left(\mathbb{A}_{\mathrm{k}}^{n}\right)_{\leq d}$.

Lemma 2.6. Let us fix some integers $d, n \geq 1$, and see $\operatorname{End}\left(\mathbb{A}_{\mathrm{k}}^{n}\right)_{\leq d}$ as an affine space, via the bijection $\operatorname{End}\left(\mathbb{A}_{\mathrm{k}}^{n}\right) \rightarrow\left(\mathrm{k}\left[x_{1}, \ldots, x_{n}\right]_{\leq d}\right)^{n}$. Then, the following hold:

(1) $J_{\mathrm{k}^{*}}=\left\{f \in \operatorname{End}\left(\mathbb{A}_{\mathrm{k}}^{n}\right)_{\leq d} \mid \operatorname{Jac}(f) \in \mathrm{k}^{*}\right\}$ is locally closed in $\operatorname{End}\left(\mathbb{A}_{\mathrm{k}}^{n}\right)_{\leq d}$, and inherits from it the structure of an affine variety.

(2) $J_{1}=\left\{f \in \operatorname{End}\left(\mathbb{A}_{\mathrm{k}}^{n}\right)_{\leq d} \mid \operatorname{Jac}(f)=1\right\}$ is closed in $\operatorname{End}\left(\mathbb{A}_{\mathrm{k}}^{n}\right)_{\leq d}$.

(3) $\operatorname{Aut}\left(\mathbb{A}_{\mathrm{k}}^{n}\right)_{\leq d}$ is a closed subset of $J_{\mathrm{K}^{*}}$.

(4) $\operatorname{SAut}\left(\mathbb{A}_{\mathrm{k}}^{n}\right)_{\leq d}$ is a closed subset of $J_{1}$.

Proof. The Jacobian being a morphism $\operatorname{End}\left(\mathbb{A}_{\mathrm{k}}^{n}\right)_{\leq d} \rightarrow \mathrm{k}\left[x_{1}, \ldots, x_{n}\right]_{\leq n(d-1)}$, the sets

$$
J_{k}=\left\{f \in \operatorname{End}\left(\mathbb{A}_{\mathrm{k}}^{n}\right)_{\leq d} \mid \operatorname{Jac}(f) \in \mathrm{k}\right\} \text { and } J_{1}
$$

are closed in $\operatorname{End}\left(\mathbb{A}_{\mathrm{k}}^{n}\right)_{\leq d}$ and are thus affine algebraic varieties. Since $J_{0}=$ $\left\{f \in \operatorname{End}\left(\mathbb{A}_{\mathrm{k}}^{n}\right)_{\leq d} \mid \operatorname{Jac}(f)=0\right\}$ is given by one equation in $J_{\mathrm{k}}$, the set $J_{\mathrm{k}^{*}}=J_{\mathrm{k}} \backslash J_{0}$ is affine (and locally closed in $\operatorname{End}\left(\mathbb{A}_{\mathrm{k}}^{n}\right)_{\leq d}$ ). This yields assertions (1) and (2).

In order to show (3) and (4), we define $W_{d}$ to be the set of nonzero $(n+1)$-tuples $\left(h_{0}, \ldots, h_{n}\right)$ of homogeneous polynomials $h_{i} \in \mathrm{k}\left[x_{0}, \ldots, x_{n}\right]$ of degree $d$, where $h_{0}=\mu x_{0}^{d}, \mu \in \mathrm{k}$, up to linear equivalence: $\left(h_{0}, \ldots, h_{n}\right) \sim\left(\lambda h_{0}, \ldots, \lambda h_{n}\right)$ for any $\lambda \in \mathrm{k}^{*}$. The equivalence class of $\left(h_{0}, \ldots, h_{n}\right)$ will be denoted by $\left[h_{0}: \cdots: h_{n}\right]$. Since the set of homogeneous polynomials of degree $d$ in $n+1$ variables is a k-vector space, this gives to $W_{d}$ a canonical projective space structure. We then denote by $B_{d} \subset W_{d}$ the hyperplane given by $h_{0}=0$ and obtain a canonical isomorphism of affine spaces

$$
\begin{aligned}
W_{d} \backslash B_{d} & \stackrel{\sim}{\longrightarrow} \operatorname{End}\left(\mathbb{A}_{\mathrm{k}}^{n}\right)_{\leq d}, \\
{\left[x_{0}^{d}: h_{1}: \cdots: h_{n}\right] } & \longmapsto\left(h_{1}\left(1, x_{1}, \ldots, x_{n}\right), \ldots, h_{n}\left(1, x_{1}, \ldots, x_{n}\right)\right) .
\end{aligned}
$$


We denote by $Y \subseteq W_{d^{n-1}} \times\left(W_{d} \backslash B_{d}\right)$ the set consisting of elements $(g, f)$, such that $h:=\left(g_{0}\left(f_{0}, \ldots, f_{n}\right), \ldots, g_{n}\left(f_{0}, \ldots, f_{n}\right)\right)$ is a multiple (maybe 0$)$ of the identity, i.e., $h_{i} x_{j}=h_{j} x_{i}$ for all $i, j$. The description of $Y$ shows that it closed in $W_{d^{n-1}} \times\left(W_{d} \backslash B_{d}\right)$. Since $W_{d^{n-1}}$ is a complete variety, the projection $p_{2}: W_{d^{n-1}} \times\left(W_{d} \backslash B_{d}\right) \rightarrow\left(W_{d} \backslash B_{d}\right)$ is a Zariski-closed morphism, so $p_{2}(Y)$ is closed in $\left(W_{d} \backslash B_{d}\right) \simeq \operatorname{End}\left(\mathbb{A}_{\mathrm{k}}^{n}\right)_{\leq d}$.

In order to show (3) and (4), we only need to show that $p_{2}(Y) \cap J_{\mathrm{k}^{*}}=\operatorname{Aut}\left(\mathbb{A}_{\mathrm{k}}^{n}\right)_{\leq d}$, which implies that $p_{2}(Y) \cap J_{1}=\operatorname{SAut}\left(\mathbb{A}_{\mathrm{k}}^{n}\right)_{\leq d}$. We then show both inclusions.

(i) If $f \in \operatorname{Aut}\left(\mathbb{A}_{\mathrm{k}}^{n}\right)_{\leq d}$, there exists $g \in \operatorname{Aut}\left(\mathbb{A}_{\mathrm{k}}^{n}\right)_{\leq d^{n-1}}$ such that $g \circ f=\mathrm{id}$ ([Bass et al. 1982, Theorem 1.5, page 292]). In consequence, we obtain $(g, f) \in Y$, so $f \in p_{2}(Y)$. The fact that $f$ belongs to $J_{\mathrm{k}^{*}}$ is given by Remark 2.3, so $\operatorname{Aut}\left(\mathbb{A}_{\mathrm{k}}^{n}\right)_{\leq d} \subseteq p_{2}(Y) \cap J_{\mathrm{k}^{*}}$. (ii) Let $(g, f) \in Y$, with $f \in J_{\mathrm{k}^{*}}$. By definition of $Y$, the element $\left(g_{0}\left(f_{0}, \ldots, f_{n}\right)\right.$, $\left.\ldots, g_{n}\left(f_{0}, \ldots, f_{n}\right)\right)$ is a multiple of the identity. There exists some $j$ such that $g_{j} \neq 0$. The fact that $f \in J_{\mathrm{k}^{*}}$ implies that $f: \mathbb{A}_{\mathrm{k}}^{n} \rightarrow \mathbb{A}_{\mathrm{k}}^{n}$ is dominant. Hence, $g_{j}\left(f_{0}\left(1, x_{0}, \ldots, x_{n}\right), \ldots, f_{n}\left(1, x_{0}, \ldots, x_{n}\right)\right)$ is not equal to zero. This implies that $g \in \operatorname{End}\left(\mathbb{A}^{n-1}\right)_{\leq d^{n-1}}$ and that $g \circ f=$ id. Hence, $f \in \operatorname{Aut}\left(\mathbb{A}_{\mathrm{k}}^{n}\right)_{\leq d}$. This yields $p_{2}(Y) \cap J_{\mathrm{k}^{*}} \subseteq \operatorname{Aut}\left(\mathbb{A}_{\mathrm{k}}^{n}\right)_{\leq d}$.

Lemma 2.7. Let us fix some integers $d, n \geq 1$, and see $\operatorname{Aut}\left(\mathbb{A}_{\mathrm{k}}^{n}\right)_{\leq d}$ and $\operatorname{SAut}\left(\mathbb{A}_{\mathrm{k}}^{n}\right)_{\leq d}$ as affine varieties, via their inclusion in $\operatorname{End}\left(\mathbb{A}_{\mathrm{k}}^{n}\right)_{\leq d} \simeq\left(\mathrm{k}\left[x_{1}, \ldots, x_{n}\right]_{\leq d}\right)^{n}$ (see Lemma 2.6). Then, the following hold:

(1) Morphisms $A \rightarrow \operatorname{Aut}\left(\mathbb{A}_{\mathrm{k}}^{n}\right)$ correspond to elements of $\operatorname{Aut}\left(\mathbb{A}_{\mathrm{k}[A]}^{n}\right)$.

(2) For each k-algebraic variety $A$, the morphisms $A \rightarrow \operatorname{Aut}\left(\mathbb{A}_{\mathrm{k}}^{n}\right)$ that have image in $\operatorname{Aut}\left(\mathbb{A}_{\mathrm{k}}^{n}\right)_{\leq d}$ correspond to morphisms of algebraic varieties $A \rightarrow \operatorname{Aut}\left(\mathbb{A}_{\mathrm{k}}^{n}\right)_{\leq d}$.

(3) The set $\operatorname{Aut}\left(\mathbb{A}_{\mathrm{k}}^{n}\right)_{\leq d}$ is closed in $\operatorname{Aut}\left(\mathbb{A}_{\mathrm{k}}^{n}\right)$, and the restriction of the topology of $\operatorname{Aut}\left(\mathbb{A}_{\mathrm{k}}^{n}\right)$ on it yields the topology of its algebraic variety structure.

(4) A subset of $\operatorname{Aut}\left(\mathbb{A}_{\mathrm{k}}^{n}\right)$ is closed if and only if its intersection with $\operatorname{Aut}\left(\mathbb{A}_{\mathrm{k}}^{n}\right)_{\leq d}$ is closed, for each integer $d \geq 1$.

Moreover, everything works the same replacing Aut with SAut.

Proof. We do the proof with Aut, the same proof works replacing Aut with SAut.

The map

$$
\operatorname{End}\left(\mathbb{A}_{\mathrm{k}}^{n}\right)_{\leq d} \times \mathbb{A}_{\mathrm{k}}^{n} \longrightarrow \operatorname{End}\left(\mathbb{A}_{\mathrm{k}}^{n}\right)_{\leq d} \times \mathbb{A}_{\mathrm{k}}^{n}, \quad(f, x) \longmapsto(f, f(x))
$$

is a morphism of algebraic varieties, so every morphism of algebraic varieties $A \rightarrow \operatorname{Aut}\left(\mathbb{A}_{\mathrm{k}}^{n}\right)_{\leq d}$ yields an $A$-automorphism of $A \times \mathbb{A}_{\mathrm{k}}^{n}$, and thus a morphism $A \rightarrow \operatorname{Aut}\left(\mathbb{A}_{\mathrm{k}}^{n}\right)$ with image in $\operatorname{Aut}\left(\mathbb{A}_{\mathrm{k}}^{n}\right)_{\leq d}$.

Conversely, let $f: A \rightarrow \operatorname{Aut}\left(\mathbb{A}_{\mathrm{k}}^{n}\right)$ be a morphism. By definition, this is given by an $A$-automorphism of $A \times \mathbb{A}_{\mathrm{k}}^{n}$. Composing with the projection $A \times \mathbb{A}_{\mathrm{k}}^{n} \rightarrow \mathbb{A}_{\mathrm{k}}^{n}$ and then 
with the function $x_{i}$ on $\mathbb{A}_{\mathrm{k}}^{n}$, we obtain an element $f_{i} \in \mathrm{k}\left[A \times \mathbb{A}_{\mathrm{k}}^{n}\right]=\mathrm{k}[A]\left[x_{1}, \ldots, x_{n}\right]$. Hence, $\left(f_{1}, \ldots, f_{n}\right)$ is an element of $\operatorname{Aut}\left(\mathbb{A}_{\mathrm{k}[A]}^{n}\right)$. Such an element yields a morphism of algebraic varieties $A \rightarrow \operatorname{End}\left(\mathbb{A}_{\mathrm{k}}^{n}\right)_{\leq m}$ for some $m \in \mathbb{N}$, having image in $\operatorname{Aut}\left(\mathbb{A}_{\mathrm{k}}^{n}\right)$. This yields (1) and (2). Assertion (3) also follows, after observing that the preimage of $\operatorname{Aut}\left(\mathbb{A}_{\mathrm{k}}^{n}\right)_{\leq i}$ is a closed subset of $A$, for each $i \geq 0$.

It remains to show (4). Let $Y \subset \operatorname{Aut}\left(\mathbb{A}_{\mathrm{k}}^{n}\right)$ be any subset. If $Y$ is closed in $\operatorname{Aut}\left(\mathbb{A}_{\mathrm{k}}^{n}\right)$, then $Y \cap \operatorname{Aut}\left(\mathbb{A}_{\mathrm{k}}^{n}\right)_{\leq d}$ is closed (in $\operatorname{Aut}\left(\mathbb{A}_{\mathrm{k}}^{n}\right)$ or $\left.\operatorname{Aut}\left(\mathbb{A}_{\mathrm{k}}^{n}\right)_{\leq d}\right)$ for each $d$, by (3). Suppose conversely that $Y \cap \operatorname{Aut}\left(\mathbb{A}_{\mathrm{k}}^{n}\right)_{\leq d}$ is closed for each $d$. In order to show that $Y$ is closed in $\operatorname{Aut}\left(\mathbb{A}_{\mathrm{k}}^{n}\right)$, we take any morphism $A \rightarrow \operatorname{Aut}\left(\mathbb{A}_{\mathrm{k}}^{n}\right)$, and show that the preimage of $Y$ is closed. As we observed before, we can see this morphism as a morphism of algebraic varieties $A \rightarrow \operatorname{Aut}\left(\mathbb{A}_{\mathrm{k}}^{n}\right)_{\leq m}$ for some $m$. Since $Y \cap \operatorname{Aut}\left(\mathbb{A}_{\mathrm{k}}^{n}\right)_{\leq m}$ is closed, the preimage of $Y$ is closed.

2B. Dynamical properties and the behaviour at infinity. In the sequel, we fix a canonical open embedding $\mathbb{A}_{\mathrm{k}}^{n} \rightarrow \mathbb{P}_{\mathrm{k}}^{n}$ given by

$$
\left(x_{1}, \ldots, x_{n}\right) \longmapsto\left[1: x_{1}: \cdots: x_{n}\right]
$$

and denote by $H_{\infty} \subset \mathbb{P}_{\mathrm{k}}^{n}$ the complement $H_{\infty}=\mathbb{P}_{\mathrm{k}}^{n} \backslash \mathbb{A}_{\mathrm{k}}^{n}$, which is a hyperplane. Once this is fixed, we have a canonical extension of any element of $\operatorname{End}\left(\mathbb{A}_{\mathrm{k}}^{n}\right)$ to a rational map $\mathbb{P}_{\mathrm{k}}^{n} \rightarrow \rightarrow \mathbb{P}_{\mathrm{k}}^{n}$. In particular, this yields inclusions

$$
\operatorname{Aut}\left(\mathbb{A}_{\mathrm{k}}^{n}\right) \longrightarrow \operatorname{Bir}\left(\mathbb{A}_{\mathrm{k}}^{n}\right) \stackrel{\simeq}{\longrightarrow} \operatorname{Bir}\left(\mathbb{P}_{\mathrm{k}}^{n}\right) .
$$

The automorphisms of degree 1 (affine automorphisms) correspond to those which extend to elements of $\operatorname{Aut}\left(\mathbb{P}_{\mathrm{k}}^{n}\right)$. The others extend to birational maps which are not automorphisms, and we can associate to them two sets, which are classical objects in dynamics (see for example [Sibony 1999; Guedj and Sibony 2002; Bisi 2008]):

Definition 2.8. For each $f \in \operatorname{Aut}\left(\mathbb{A}_{\mathrm{k}}^{n}\right)$ of degree $\geq 2$, we define $I_{f} \subset \mathbb{P}_{\mathrm{k}}^{n}$ to be the indeterminacy locus of the extension of $f$ to $\mathbb{P}_{\mathrm{k}}^{n}$, and $X_{f} \subset \mathbb{P}_{\mathrm{k}}^{n}$ to be the image of $H_{\infty} \backslash\left(I_{f}\right)$.

In order to see the sets $I_{f}, X_{f}$ explicitly, we use the following definition.

Definition 2.9. If $f=\left(f_{1}, \ldots, f_{n}\right) \in \operatorname{Aut}\left(\mathbb{A}_{\mathrm{k}}^{n}\right)$ is an element of degree $d$ and $a_{i}$ is the homogeneous part of $f_{i}$ of degree $d$, for $i=1, \ldots, n$, we say that $\left(a_{1}, \ldots, a_{n}\right) \in$ $\operatorname{End}\left(\mathbb{A}_{\mathrm{k}}^{n}\right)$ is the highest homogeneous part of $f$.

Remark 2.10. If $\left(a_{1}, \ldots, a_{n}\right)$ is the highest homogeneous part of $f \in \operatorname{Aut}\left(\mathbb{A}_{\mathrm{k}}^{n}\right)$, the set $I_{f} \subset \mathbb{P}_{\mathrm{k}}^{n}$ is given by

$$
x_{0}=0, \quad a_{1}=\cdots=a_{n}=0,
$$

and is a proper closed subset of the hyperplane at infinity $H_{\infty}$ (given by $x_{0}=0$ ). 
Moreover, the set $X_{f}$ is equal to

$$
\left\{\left[0: a_{1}\left(x_{1}, \ldots, x_{n}\right): \cdots: a_{n}\left(x_{1}, \ldots, x_{n}\right)\right] \mid\left[0: x_{1}: \cdots: x_{n}\right] \in H_{\infty} \backslash I_{f}\right\} .
$$

Remark 2.11 (regular terminology). In dynamics, the elements $f \in \operatorname{Aut}\left(\mathbb{A}_{\mathrm{k}}^{n}\right)$ such that $I_{f} \cap I_{f^{-1}}=\varnothing$ are called "regular" and elements such that $X_{f} \cap I_{f}=\varnothing$ are called "weakly regular" in [Guedj and Sibony 2002] or "quasiregular" in [Bisi 2008]. The use of this terminology in algebraic geometry can be confusing, because of the common use of the word regular for maps (in fact all elements of $\operatorname{Aut}\left(\mathbb{A}_{\mathrm{k}}^{n}\right)$ are morphisms, hence biregular). This is why we will say "dynamically regular" if $I_{f} \cap I_{f^{-1}}=\varnothing$; we will not use the words "quasiregular" or "weakly regular".

Remark 2.12. Fixing the degree $d$, we obtain an algebraic variety $\operatorname{Aut}\left(\mathbb{A}_{\mathrm{k}}^{n}\right)_{d}$. It follows from the definition that the set of dynamically regular elements of $\operatorname{Aut}\left(\mathbb{A}_{\mathrm{k}}^{n}\right)_{d}$ is an open subset (in general not dense). However, the set of dynamically regular elements of $\operatorname{Aut}\left(\mathbb{A}_{\mathrm{k}}^{n}\right)_{\leq d}$ (and thus of $\operatorname{Aut}\left(\mathbb{A}_{\mathrm{k}}^{n}\right)$ ) is not open in general. For $n=2$, this can be seen by taking for example

$$
f=\left(-x_{2}, x_{1}+x_{2}^{2}\right) \in \operatorname{SAut}\left(\mathbb{A}_{\mathrm{k}}^{2}\right), \quad \alpha=\left(x_{1}+t x_{2}^{2}, x_{2}\right) \in \operatorname{SAut}\left(\mathbb{A}_{\mathrm{k}[t]}^{2}\right),
$$

and considering the family $\beta=\alpha f \alpha^{-1} \in \operatorname{SAut}\left(\mathbb{A}_{\mathrm{k}[t]}^{2}\right)$. Then, $\beta(t)$ is not dynamically regular for $t \in \mathrm{k}^{*}$ but $\beta(0)=f$ is dynamically regular.

Remark 2.13. If $n=2$, we find $X_{f}=I_{f^{-1}}$. Indeed, the extension of an element $f \in \operatorname{Aut}\left(\mathbb{A}_{\mathrm{k}}^{2}\right)$ of degree $>1$ is an element of $\operatorname{Bir}\left(\mathbb{P}_{\mathrm{k}}^{2}\right)$ which contracts the line at infinity onto one point $X_{f}$. The inverse of this birational map is then defined at any other point of $H_{\infty}$, so we find $X_{f}=I_{f^{-1}}$.

We now show that the degree of a composition is determined by the sets $I_{f}$ and $X_{f}$ defined above. The following results are classical in the world of dynamics, we recall the easy proofs for self-containedness.

Lemma 2.14. Let $f, g \in \operatorname{Aut}\left(\mathbb{A}_{\mathrm{k}}^{n}\right)$ be of degree $\geq 2$. Then, the following are equivalent:

(1) $\operatorname{deg}(g f)=\operatorname{deg}(g) \cdot \operatorname{deg}(f)$.

(2) The set $X_{f}$ is not contained in $I_{g}$.

Moreover, if both conditions hold, then $X_{g f} \subset X_{g}$.

Proof. Denoting by $\left(a_{1}, \ldots, a_{n}\right)$ and $\left(b_{1}, \ldots, b_{n}\right)$ the highest homogeneous parts of $f$ and $g$ respectively, the equality $\operatorname{deg}(g f)=\operatorname{deg}(g) \cdot \operatorname{deg}(f)$ is equivalent to the fact that one of the polynomials

$$
b_{1}\left(a_{1}, \ldots, a_{n}\right), \ldots, b_{n}\left(a_{1}, \ldots, a_{n}\right)
$$

is not equal to zero. 
By definition, the sets $I_{g}, X_{f} \subset H_{\infty}$ are given respectively by

$$
\begin{aligned}
I_{g} & =\left\{\left[0: y_{1}: \cdots: y_{n}\right] \in H_{\infty} \mid b_{1}\left(y_{1}, \ldots, y_{n}\right)=\cdots=b_{n}\left(y_{1}, \ldots, y_{n}\right)=0\right\}, \\
X_{f} & =\left\{\left[0: a_{1}\left(y_{1}, \ldots, y_{n}\right): \cdots: a_{n}\left(y_{1}, \ldots, y_{n}\right)\right] \mid\left[0: y_{1}: \cdots: y_{n}\right] \in H_{\infty} \backslash I_{f}\right\} .
\end{aligned}
$$

If $X_{f} \not \subset I_{g}$, then there exists a point $\left[0: y_{1}: \cdots: y_{n}\right] \in H_{\infty} \backslash I_{f}$ such that $\left[0: a_{1}\left(y_{1}, \ldots, y_{n}\right): \cdots: a_{n}\left(y_{1}, \ldots, y_{n}\right)\right] \notin I_{g}$, which corresponds to the existence of an index $i \in\{1, \ldots, n\}$ such that $b_{i}\left(a_{1}\left(y_{1}, \ldots, y_{n}\right), \ldots, a_{n}\left(y_{1}, \ldots, y_{n}\right)\right) \neq 0$. In particular, the polynomial $b_{i}\left(a_{1}, \ldots, a_{n}\right)$ is not zero, so $\operatorname{deg}(g f)=\operatorname{deg}(g) \operatorname{deg}(f)$.

Conversely, if $b_{i}\left(a_{1}, \ldots, a_{n}\right)$ is a nonzero polynomial, the open subset $U_{i} \subset H_{\infty}$ corresponding to the nonvanishing of this polynomial is nonempty. Intersecting this open set with $H_{\infty} \backslash I_{f}$ yields a nonempty open subset of points in $H_{\infty}$ which have image in $X_{f}$ and not in $I_{g}$.

Now that the equivalence between (1) and (2) is shown, we show that these imply that $X_{g f} \subset X_{g}$. Since $\operatorname{deg}(g f)=\operatorname{deg}(g) \operatorname{deg}(f)$, the homogeneous part of highest degree of $g f$ is $\left(b_{1}\left(a_{1}, \ldots, a_{n}\right), \ldots, b_{n}\left(a_{1}, \ldots, a_{n}\right)\right)$. This implies that the points of $X_{g f}$ are in the image by $g$ (or more precisely of its extension to $\mathbb{P}_{\mathrm{k}}^{n}$ ) of the set $X_{f} \backslash I_{g}$, and thus lie in $X_{g}$.

Corollary 2.15. Let $f, g \in \operatorname{Aut}\left(\mathbb{A}_{\mathrm{k}}^{n}\right)$ be of degree $\geq 2$. Then, the following hold:

(1) $X_{f} \subset I_{f^{-1}}$.

(2) If $I_{g} \cap I_{f^{-1}}=\varnothing$, then $\operatorname{deg}(g f)=\operatorname{deg}(g) \cdot \operatorname{deg}(f)$.

(3) $X_{f} \not \subset I_{f} \Leftrightarrow \operatorname{deg}\left(f^{2}\right)=\operatorname{deg}(f)^{2}$.

Proof. Since $\operatorname{deg}\left(f^{-1} f\right)<\operatorname{deg}\left(f^{-1}\right) \operatorname{deg}(f)$, part (1) follows from Lemma 2.14. If $I_{g} \cap I_{f^{-1}}=\varnothing$, then $X_{f} \not \subset I_{g}$ by (1), so the equality $\operatorname{deg}(g f)=\operatorname{deg}(g) \operatorname{deg}(f)$ follows again from Lemma 2.14. Part (3) corresponds to Lemma 2.14, in the case $f=g$.

Corollary 2.16. If $f \in \operatorname{Aut}\left(\mathbb{A}_{\mathrm{k}}^{n}\right)$ is an element such that $X_{f} \cap I_{f}=\varnothing$, then

$$
\operatorname{deg}\left(f^{m}\right)=\operatorname{deg}(f)^{m} \quad \text { and } \quad X_{f^{m}} \subset X_{f}
$$

for each $m \geq 1$.

Proof. We prove the result by induction on $m$, the case $m=1$ being obvious. For $m \geq 2$, we use the facts that $X_{f} \cap I_{f}=\varnothing$ and $X_{f^{m-1}} \subset X_{f}$, which imply that $X_{f^{m-1}} \not \subset I_{f}$. Applying Lemma 2.14 to the composition $f \circ f^{m-1}$, we obtain that $\operatorname{deg}\left(f^{m}\right)=\operatorname{deg}\left(f^{m-1}\right) \operatorname{deg}(f)$, which is equal to $\operatorname{deg}(f)^{m}$ by the induction hypothesis, and also that $X_{f^{m}} \subset X_{f}$.

Restricting to dimension 2, we obtain the following result.

Corollary 2.17. Let $f \in \operatorname{Aut}\left(\mathbb{A}_{\mathrm{k}}^{2}\right)$ be of degree $\geq 1$. The following are equivalent:

(1) $I_{f} \neq I_{f^{-1}}$; 
(2) $I_{f} \cap I_{f^{-1}}=\varnothing$;

(3) $\operatorname{deg}\left(f^{2}\right)=\operatorname{deg}(f)^{2}$;

(4) $\operatorname{deg}\left(f^{m}\right)=\operatorname{deg}(f)^{m}$ for each $m \geq 1$.

Proof. As we are in dimension 2, we have $X_{f}=I_{f^{-1}}$ and $X_{f^{-1}}=I_{f}$, which are two points of $H_{\infty}$, which can be distinct or not (see Remark 2.13).

The equality $\operatorname{deg}\left(f^{2}\right)=\operatorname{deg}(f)^{2}$ is equivalent to $X_{f} \not \subset I_{f}$ (Corollary 2.15), which is here equivalent to $I_{f^{-1}} \neq I_{f}$ or $I_{f^{-1}} \cap I_{f}=\varnothing$. Hence, (1), (2), and (3) are equivalent, and of course implied by (4). It remains to see that (2) corresponds to $X_{f} \cap I_{f}=\varnothing$, which implies that $\operatorname{deg}\left(f^{m}\right)=\operatorname{deg}(f)^{m}$ for each $m \geq 1$ by Corollary 2.16 .

Remark 2.18. The most interesting implication of this corollary is $(3) \Rightarrow(4)$, i.e., that $\operatorname{deg}\left(f^{2}\right)=\operatorname{deg}(f)^{2}$ implies that $\operatorname{deg}\left(f^{m}\right)=\operatorname{deg}(f)^{m}$ for $m \geq 1$, a fact already observed by Jean-Philippe Furter [1999] (at least when $\operatorname{char}(\mathrm{k})=0$ ).

Looking at the proof, this result has no reason to be true in dimension $n \geq 3$, and is in fact false on the easiest nontrivial example, that we describe now.

Example 2.19. Let $f=\left(x_{1}+x_{2}^{2}, x_{2}+x_{3}^{2}, x_{3}\right) \in \operatorname{SAut}\left(\mathbb{A}_{\mathrm{k}}^{3}\right)$, which has highest homogeneous part $a=\left(x_{2}^{2}, x_{3}^{2}, 0\right) \in \operatorname{End}\left(\mathbb{A}_{\mathrm{k}}^{3}\right)$. Then,

$$
I_{f}=[0: 1: 0: 0], \quad X_{f}=\left\{\left[0: x_{1}: x_{2}: 0\right] \mid\left[x_{1}: x_{2}\right] \in \mathbb{P}^{1}\right\} .
$$

Since $X_{f} \not \subset I_{f}$, we have $\operatorname{deg}\left(f^{2}\right)=\operatorname{deg}(f)^{2}=4$ and $X_{f^{2}} \subset X_{f}$. More precisely, the homogeneous part of $f^{2}$ is $a^{2}=\left(x_{3}^{4}, 0,0\right)$, so

$$
I_{f^{2}}=I_{f}=X_{f^{2}}=[0: 1: 0: 0] \text {. }
$$

In particular, $X_{f^{2}} \subset I_{f}$, so $\operatorname{deg}\left(f^{3}\right)<\operatorname{deg}\left(f^{2}\right) \cdot \operatorname{deg}(f)$.

In fact, one easily checks with the formulas that $\operatorname{deg}\left(f^{n}\right) \leq 4$ for each $n$, and that $\operatorname{deg}\left(f^{n}\right)=4$ for each $n \geq 2$ if $\operatorname{char}(\mathrm{k})=0$. Indeed

$$
f^{n}=\left(x_{1}+n x_{2}^{2}+n(n-1) x_{2} x_{3}^{2}+\left(\sum_{i=1}^{n-1} i^{2}\right) x_{3}^{4}, x_{2}+n x_{3}^{2}, x_{3}\right),
$$

for each $n \geq 0$.

2C. Families of automorphisms and valuations. In the sequel, we will study families of elements of $\operatorname{Aut}\left(\mathbb{A}_{\mathrm{k}}^{n}\right)$, which correspond to elements of $\operatorname{Aut}\left(\mathbb{A}_{\mathrm{k}((t))}^{n}\right)$.

It is then natural to use the valuation

$$
v: \mathrm{k}((t))\left[x_{1}, \ldots, x_{n}\right] \rightarrow \mathbb{Z} \cup\{-\infty\}
$$

associated to $t$. We define precisely this valuation here, as we will use it often afterwards. 
Definition 2.20. Every element $f \in \mathrm{k}((t))\left[x_{1}, \ldots, x_{n}\right] \backslash\{0\}$ can be written as

$$
f=\sum_{k=m}^{\infty} a_{k} t^{k}
$$

where $m \in \mathbb{Z}, a_{i} \in \mathrm{k}\left[x_{1}, \ldots, x_{n}\right]$ for $i \geq m$, and $a_{m} \neq 0$. We then define $v(f)=m$. Choosing $v(0)=-\infty$, we obtain a valuation

$$
v: \mathrm{k}((t))\left[x_{1}, \ldots, x_{n}\right] \longrightarrow \mathbb{Z} \cup\{-\infty\} .
$$

(1) If $v(f)=0$, we define $f(0)=a_{0}$.

(2) If $v(f)>0$, we define $f(0)=0$.

(3) If $v(f)<0$, we say that $f$ has a pole at $t=0$ and that $f(0)$ is not defined.

Definition 2.21. Let $f=\left(f_{1}, \ldots, f_{n}\right) \in \operatorname{Aut}\left(\mathbb{A}_{\mathrm{k}((t))}^{n}\right)$. We define

$$
v(f)=\min \left\{v\left(f_{i}\right) \mid i=1, \ldots, n\right\} .
$$

(1) If $v(f) \geq 0$, we say that $f$ is defined at the origin (or has a value), and define

$$
f(0)=\left(f_{1}(0), \ldots, f_{n}(0)\right)
$$

to be its value, which is an element of $\operatorname{End}\left(\mathbb{A}_{\mathrm{k}}^{n}\right)$.

(2) If $v(f)<0$, we say that $f$ has a pole at $t=0$, and say that $f(0)$ is not defined.

Remark 2.22. In the above definition, it is possible that the element $f(0)$ is defined, but does not belong to $\operatorname{Aut}\left(\mathbb{A}_{\mathrm{k}}^{n}\right)$. This is for example the case when one of the components becomes 0 , or for $f=\left(t x_{1}+x_{2}, x_{2}, \ldots, x_{n}\right)$. This phenomenon is however impossible for $\operatorname{SAut}\left(\mathbb{A}_{\mathrm{k}}^{n}\right)$, as the following result shows.

Lemma 2.23. Let $\alpha \in \mathrm{SAut}\left(\mathbb{A}_{\mathrm{k}((t))}^{n}\right)$ be an element which has no pole at $t=0$.

Then, $\alpha^{-1}$ has no pole at $t=0$, and replacing $t$ with 0 yields two automorphisms $\beta, \gamma \in \operatorname{SAut}\left(\mathbb{A}_{\mathrm{k}}^{n}\right)$

$$
\beta=\alpha(0), \quad \gamma=\alpha^{-1}(0)
$$

such that $\beta \gamma=\mathrm{id}$.

Proof. Since $\alpha$ has no pole at $t=0$, it sends $(0, \ldots, 0) \in \mathbb{A}_{\mathrm{k}((t))}^{n}$ onto an element having coordinates in $\mathrm{k} \llbracket t \rrbracket$. We can thus replace $\alpha$ by its composition with a translation and assume that $\alpha$ (and thus $\alpha^{-1}$ ) fixes the origin. Its linear part is then equal to an element of $\operatorname{SL}(n, \mathrm{k} \llbracket t \rrbracket)$, whose inverse also belongs to $\operatorname{SL}(n, \mathrm{k} \llbracket t \rrbracket)$. Replacing with the composition by this inverse, we can assume that $\alpha$ has a trivial linear part. We denote by $\mathfrak{m}$ the ideal of $\mathrm{k}((t))\left[x_{1}, \ldots, x_{n}\right]$ generated by the $x_{i}$, and can then write

$$
\alpha=\left(f_{1}, \ldots, f_{n}\right)
$$


for some $f_{1}, \ldots, f_{n} \in \mathrm{k} \llbracket t \rrbracket\left[x_{1}, \ldots, x_{n}\right], f_{i} \equiv x_{i}\left(\bmod \mathfrak{m}^{2}\right)$. We write then

$$
\alpha^{-1}=\left(x_{1}+\sum_{i=2}^{d} g_{i, 1}, \ldots, x_{n}+\sum_{i=2}^{d_{2}} g_{i, n}\right)
$$

where the $g_{i, j} \in \mathrm{k}((t))\left[x_{1}, \ldots, x_{n}\right]$ are homogeneous polynomials of degree $i$ and $d_{2}$ is the degree of $g$. Assume for contradiction that one of the $g_{k, j}$ does not belong to $\mathrm{k} \llbracket t \rrbracket\left[x_{1}, \ldots, x_{n}\right]$, and choose $k$ to be minimal for this. Then, the $j$-th coordinate of $\alpha^{-1} \circ \alpha$ is equal to

$$
x_{j}=f_{j}+\sum_{i=2}^{d_{2}} g_{i, j}\left(f_{1}, \ldots, f_{n}\right),
$$

which implies that $\sum_{i=k}^{d} g_{i, j}\left(f_{1}, \ldots, f_{n}\right) \in \mathrm{k} \llbracket t \rrbracket\left[x_{1}, \ldots, x_{n}\right]$. But the part of degree $k$ of this sum is in fact equal to $g_{i, j}\left(x_{1}, \ldots, x_{n}\right)$, which does not belong to $\mathrm{k} \llbracket t]\left[x_{1}, \ldots, x_{n}\right]$.

We have proved that $\alpha^{-1}$ does not have any pole at the origin. We then denote by $d_{1}, d_{2}$ the degrees of $\alpha$ and $\alpha^{-1}$ (which are the maximal degree of their components), and denote by $\operatorname{End}_{d_{i}}$ the set of endomorphisms of $\mathbb{A}^{n}$ of degree $\leq d_{i}$, which is naturally isomorphic to an affine space. Observe that $\left(\alpha, \alpha^{-1}\right)$ corresponds to a $\mathrm{k}((t))$-point of the algebraic variety

$$
\left\{(f, g) \in \operatorname{End}_{d_{1}} \times \operatorname{End}_{d_{1}} \mid f \circ g=\mathrm{id}\right\} .
$$

Since neither $\alpha$ neither $\alpha^{-1}$ has a pole at $t=0$, all coefficients are defined at the origin. Replacing $t$ with 0 gives then the result.

Remark 2.24. The result of Lemma 2.23 can also be obtained from the fact that each $\operatorname{SAut}\left(\mathbb{A}_{\mathrm{k}}^{n}\right)_{\leq d}$ is closed in $\operatorname{End}\left(\mathbb{A}_{\mathrm{k}}^{n}\right)_{\leq d}($ Lemma 2.6).

We will apply a classical valuative result (Lemma 2.25 below), and recall the argument of the proof, given in [Furter 2009, §1.2] (the version that we need here is slightly more general, but the proof is analogous).

Lemma 2.25. Let $Y, Z$ be two quasiprojective k-algebraic varieties, let $\varphi: Y \rightarrow Z$ be a morphism and let $z \in Z$ be a (closed) point of $Z$. The following assertions are equivalent:

(1) The point $z$ belongs to the closure $\overline{\varphi(Y)}$ of the image.

(2) There is an irreducible $\mathrm{k}$-curve $\Gamma$, a smooth closed point $p \in \Gamma$ and a rational map $\iota: \Gamma \rightarrow Y$ such that $\varphi \circ \iota: \Gamma \rightarrow Z$ is defined at $p$ and sends it onto $z$.

(3) There is a $\mathrm{k}((t))$-point $y \in Y(\mathrm{k}((t)))$ such that $\varphi(y) \in Z(\mathrm{k}((t)))$ has no pole at $t=0$ and $\varphi(y)(0)=z$. 
Proof. (1) $\Rightarrow(2)$ : Replacing $Y$ with one of its irreducible components, we can assume that $Y$ is irreducible. Putting $Z$ into a projective variety, we can assume that $Z$ is projective. Then, replacing $Z$ with $\overline{\varphi(Y)}$ we can assume that $\varphi$ is dominant. Since $\varphi(Y)$ is an irreducible constructible subset of $Z$, it contains a dense open subset $U$ of $Z$. If $Z$ is a point, then $\varphi(Y)=Z$, in which case (2) is obvious, so we can assume that $\operatorname{dim} Z \geq 1$, and can take a closed irreducible curve $C \subset Z$ containing $z$ and meeting $U$ (through every two points passes at least one irreducible closed curve, see [Mumford 1970, §II.6, Lemma, page 56]).

We then denote by $Y^{\prime} \subset Y$ an irreducible component of $\varphi^{-1}(C)$ such that the restriction of $\varphi$ yields a dominant morphism $Y^{\prime} \rightarrow C$. We choose two points $p_{1}, p_{2} \in Y^{\prime}$ such that $\varphi\left(p_{1}\right) \neq \varphi\left(p_{2}\right)$ and take an irreducible curve $\Gamma_{0} \subset Y^{\prime}$ passing through $p_{1}, p_{2}$ (using again the lemma from [Mumford 1970]). The restriction of $\varphi$ to $\Gamma_{0}$ yields a dominant morphism $\Gamma_{0} \rightarrow C$. We can take an open embedding $v: \Gamma_{0} \rightarrow \bar{\Gamma}_{0}$, where $\bar{\Gamma}_{0}$ is an irreducible projective, and denote by $\eta: \Gamma \rightarrow \bar{\Gamma}_{0}$ the normalisation. Then, $\varphi \circ v^{-1} \circ \eta$ yields a rational map $\Gamma \rightarrow C$, which is a surjective morphism. It remains to choose for $\iota: \Gamma \rightarrow \Gamma_{0} \subset Y^{\prime} \subset Y$ the rational map $v^{-1} \circ \eta$.

(2) $\Rightarrow$ (3): The rational maps $\Gamma-\rightarrow Y \rightarrow Z$ correspond to field homomorphisms $\mathrm{k}(Z) \rightarrow \mathrm{k}(Y) \rightarrow \mathrm{k}(\Gamma)$, sending the local ring $\mathcal{O}_{z, Z}$ to $\mathcal{O}_{p, \Gamma}$.

Denote by $\widehat{\mathcal{O}}_{p, \Gamma}$ the completion of $\mathcal{O}_{p, \Gamma}$, with respect to its maximal ideal. Because $\mathcal{O}_{p, \Gamma}$ is a Noetherian regular local ring of dimension 1 with residue field $\mathrm{k}$, its completion is a complete Noetherian regular local ring with the same properties, and by the Cohen theorem, it must be isomorphic to a ring of formal power series. The dimension being 1 , one has a $\mathrm{k}$-isomorphism $\widehat{\mathcal{O}}_{p, \Gamma} \simeq \mathrm{k} \llbracket t \rrbracket$, which induces a field homomorphism $\mathrm{k}(\Gamma) \rightarrow \mathrm{k}((t))$.

The composition $\mathrm{k}(Y) \rightarrow \mathrm{k}(\Gamma) \rightarrow \mathrm{k}((t))$ corresponds to the $\mathrm{k}((t))$-point $y$ that we want, and its image corresponds to the composition $\mathrm{k}(Z) \rightarrow \mathrm{k}(Y) \rightarrow \mathrm{k}(\Gamma) \rightarrow \mathrm{k}((t))$.

$(3) \Rightarrow(1)$ : View $Z$ as a locally closed subset of $\mathbb{P}^{n}$ and take a polynomial equation $F \in \mathrm{k}\left[x_{0}, \ldots, x_{n}\right]$ that vanishes on $\varphi(Y)$. Since $\varphi(y)$ is a $\mathrm{k}((t))$-point of $\varphi(Y)$, we have $F(\varphi(y))=0$. Replacing $t$ with 0 we obtain $F(z)=0$. This shows that $z$ belongs to the closure of $Z$.

Corollary 2.26. Let $f \in \operatorname{SAut}\left(\mathbb{A}_{\mathrm{k}}^{n}\right)$, let $d \geq 1$ be an integer and let $Y$ be the $\mathrm{k}$ algebraic variety $\operatorname{SAut}\left(\mathbb{A}_{\mathrm{k}}^{n}\right)_{\leq d}$. The following assertions are equivalent:

(1) The set $\left\{g f g^{-1} \mid g \in Y\right\}$ is closed in $\operatorname{SAut}\left(\mathbb{A}_{\mathrm{k}}^{n}\right)$.

(2) If $\Gamma$ is an irreducible $\mathrm{k}-c u r v e ~ \Gamma$ and $\iota: \Gamma \rightarrow Y$ is a rational map such that $\varphi \circ \iota: \Gamma \rightarrow Z$ is defined at a smooth point $p \in \Gamma$, the image of $p$ belongs to $\left\{g f g^{-1} \mid g \in Y\right\}$.

(3) If $\varphi \in Y(\mathrm{k}((t)))$ is an element that has poles at $t=0$ and $h=\varphi f \varphi^{-1}$ has no poles at $t=0$, then $h(0)$ belongs to $\left\{g \mathrm{fg}^{-1} \mid g \in Y\right\}$. 
Proof. The degree of the inverse of an element $g \in Y$ is at most $d^{n-1}$ (see [Bass et al. 1982, Theorem 1.5, page 292]). Hence, the map $Y \rightarrow \operatorname{SAut}\left(\mathbb{A}_{\mathrm{k}}^{n}\right)$ that sends $g$ onto $g f g^{-1}$ corresponds to a morphism of algebraic varieties $\varphi: Y \rightarrow \operatorname{SAut}\left(\mathbb{A}_{\mathrm{k}}^{n}\right)_{\leq m}$ for some $m$. The result follows then from Lemma 2.25 applied to $\varphi$, and Lemma 2.7.

\section{Conjugacy classes of dynamically regular automorphisms of $\mathbb{A}_{k}^{n}$}

3A. Image at infinity of elements of $\operatorname{Aut}\left(\mathbb{A}_{\mathbf{k}((t)))}^{\boldsymbol{n}}\right)$. In Section $2 \mathrm{~B}$, we explained how the set $X_{f} \subset H_{\infty}$ is defined, for each element $f \in \operatorname{Aut}\left(\mathbb{A}_{\mathrm{k}}^{n}\right)$, by extending the map to $\mathbb{P}_{\mathrm{k}}^{n}$ and looking at the image of the hyperplane $H_{\infty}$ at infinity.

We now associate similarly a subset $X_{\alpha} \subset H_{\infty}$ to an element $\alpha \in \operatorname{Aut}\left(\mathbb{A}_{\mathrm{k}((t))}^{n}\right)$ which has a pole at $t=0$, by taking the limit of the image of $\alpha(t)$, when $t$ goes towards 0 . The formal definition of $X_{\alpha}$ is the following:

Definition 3.1. Let $\alpha \in \operatorname{Aut}\left(\mathbb{A}_{\mathrm{k}((t))}^{n}\right)$ be an element of valuation $v(\alpha)=-m<0$, that we write

$$
\alpha=\left(\frac{1}{t^{m}} \alpha_{1}, \ldots, \frac{1}{t^{m}} \alpha_{n}\right),
$$

where $\alpha_{1}, \ldots, \alpha_{n} \in \mathrm{k} \llbracket t \rrbracket\left[x_{1}, \ldots, x_{n}\right]$ are such that

$$
\tilde{\alpha}=\left(\alpha_{1}(0), \ldots, \alpha_{n}(0)\right)=\left(\tilde{\alpha}_{1}, \ldots, \tilde{\alpha}_{n}\right) \in \operatorname{End}\left(\mathbb{A}_{\mathrm{k}}^{n}\right) \backslash\{0\} .
$$

We then define

$$
X_{\alpha}=\left\{\left[0: \tilde{\alpha}_{1}\left(y_{1}, \ldots, y_{n}\right): \cdots: \tilde{\alpha}_{n}\left(y_{1}, \ldots, y_{n}\right)\right] \mid\left(y_{1}, \ldots, y_{n}\right) \in \mathbb{A}_{\mathrm{k}}^{n} \backslash \tilde{\alpha}^{-1}(\{0\})\right\} \subset H_{\infty} .
$$

This definition can be geometrically understood:

Remark 3.2. In the above definition, $\alpha$ is not defined at $t=0$, but extending $\alpha$ to $\mathbb{P}^{n}$ we obtain the element of $\operatorname{Bir}\left(\mathbb{P}_{\mathrm{k}((t))}^{n}\right)$ given by

$$
\left[x_{0}: \cdots: x_{n}\right] \rightarrow\left[t^{m} x_{0}^{d}: F_{1}\left(x_{0}, \ldots, x_{n}, t\right): \cdots: F_{n}\left(x_{0}, \ldots, x_{n}, t\right)\right],
$$

where $d$ is the degree of $\alpha$ and each $F_{i}\left(x_{0}, \ldots, x_{n}, t\right) \in \mathrm{k} \llbracket t \rrbracket\left[x_{0}, \ldots, x_{n}\right]$ is the homogenisation of $\alpha_{i}$. This corresponds to a family of rational maps of $\mathbb{P}_{\mathrm{k}}^{n}$ parametrised by $t$, which has a value at $t=0$, corresponding to

$$
\left[x_{0}: \cdots: x_{n}\right] \rightarrow\left[0: F_{1}\left(x_{0}, \ldots, x_{n}, 0\right): \cdots: F_{n}\left(x_{0}, \ldots, x_{n}, 0\right)\right] .
$$

The set $X_{\alpha} \subset H_{\infty}$ is then the image of this map by points of $\mathbb{A}_{\mathrm{k}}^{n}$ which are welldefined under this map.

Writing such a point as $\left[1: x_{1}: \cdots: x_{n}\right]$, its image by the extension of $\alpha$ is

$$
\begin{aligned}
{\left[t^{m}: F_{1}\left(1, \ldots, x_{n}, t\right): \cdots: F_{n}\left(1, \ldots, x_{n}, t\right)\right] } & \\
& =\left[t^{m}: \alpha_{1}\left(x_{1}, \ldots, x_{n}, t\right): \cdots: \alpha_{n}\left(x_{1}, \ldots, x_{n}, t\right)\right]
\end{aligned}
$$


and corresponds to a curve in $\mathbb{P}^{n}$ whose point, when $t=0$, belongs to $X_{\alpha}$.

The set $X_{\alpha}$ corresponds then to the limit, viewed in $\mathbb{P}_{\mathrm{k}}^{n}$, of the image of points of $\mathbb{A}_{\mathrm{k}}^{n}$ under $\alpha(t)$ when $t$ goes towards 0 .

Proposition 3.3. Let $f \in \operatorname{SAut}\left(\mathbb{A}_{\mathrm{k}}^{n}\right)$ be an element of degree $d>1$, and let $\alpha \in$ $\operatorname{SAut}\left(\mathbb{A}_{\mathrm{k}((t))}^{n}\right)$ be an element that has a pole at $t=0$. If

$$
X_{\alpha} \not \subset I_{f}
$$

then $\alpha^{-1} f \alpha$ has a pole at $t=0$.

Proof. We write

$$
f=\left(f_{1}, \ldots, f_{n}\right)
$$

for some $f_{1}, \ldots, f_{n} \in \mathrm{k}\left[x_{1}, \ldots, x_{n}\right]$ and denote by $\left(a_{1}, \ldots, a_{n}\right)$ the highest homogeneous part of $f$ (which is of degree $d$ ). In particular, the indeterminacy locus $I_{f} \subset \mathbb{P}_{\mathrm{k}}^{n}$ of the extension of $f$ to $\mathbb{P}_{\mathrm{k}}^{n}$ is given by

$$
x_{0}=0, \quad a_{1}=\cdots=a_{n}=0 .
$$

Because $\alpha$ has a pole at $t=0$, we have $v(\alpha)=-m<0$ and can write $\alpha=$ $\left(t^{-m} \alpha_{1}, \ldots, t^{-m} \alpha_{n}\right)$, where $\alpha_{1}, \ldots, \alpha_{n} \in \mathrm{k} \llbracket t \rrbracket\left[x_{1}, \ldots, x_{n}\right]$ are such that

$$
\tilde{\alpha}=\left(\alpha_{1}(0), \ldots, \alpha_{n}(0)\right)=\left(\tilde{\alpha}_{1}, \ldots, \tilde{\alpha}_{n}\right) \in \operatorname{End}\left(\mathbb{A}_{\mathrm{k}}^{n}\right) \backslash\{0\},
$$

and so the subset $X_{\alpha} \subset H_{\infty}$ is described by

$$
X_{\alpha}=\left\{\left[0: \tilde{\alpha}_{1}\left(y_{1}, \ldots, y_{n}\right): \ldots: \tilde{\alpha}_{n}\left(y_{1}, \ldots, y_{n}\right)\right] \mid\left(y_{1}, \ldots, y_{n}\right) \in \mathbb{A}_{\mathrm{k}}^{n} \backslash \tilde{\alpha}^{-1}(\{0\})\right\} .
$$

The fact that $X_{\alpha}$ is not included in $I_{f}$ yields a point $\left(y_{1}, \ldots, y_{n}\right) \in \mathbb{A}_{\mathrm{k}}^{n} \backslash \tilde{\alpha}^{-1}(\{0\})$ and an integer $i \in\{1, \ldots, n\}$ such that

$$
a_{i}\left(\tilde{\alpha}_{1}\left(y_{1}, \ldots, y_{n}\right), \ldots, \tilde{\alpha}_{n}\left(y_{1}, \ldots, y_{n}\right)\right) \neq 0 \text {. }
$$

In particular, we have

$$
a_{i}\left(\tilde{\alpha}_{1}, \ldots, \tilde{\alpha}_{n}\right)=a_{i}\left(\alpha_{1}(0), \ldots, \alpha_{n}(0)\right) \in \mathrm{k}\left[x_{1}, \ldots, x_{n}\right] \backslash\{0\} .
$$

Thus $a_{i}\left(t^{-m} \alpha_{1}, \ldots, t^{-m} \alpha_{n}\right)=t^{-m d} a_{i}\left(\alpha_{1}, \ldots, \alpha_{n}\right)$ has valuation $-m d$, and hence $f \alpha$ has also valuation $-m d$.

It remains to show that this implies that $\beta=\alpha^{-1} f \alpha$ has a pole at $t=0$. Indeed, if $\beta$ had no pole, we would have $v(\alpha \beta) \geq v(\alpha)=-m$, which is impossible since $f \alpha=\alpha \beta$ and $v(f \alpha)=-m d$.

Corollary 3.4. Let $f \in \operatorname{SAut}\left(\mathbb{A}_{\mathrm{k}}^{n}\right)$ be a dynamically regular element.

(1) If $\alpha \in \operatorname{SAut}\left(\mathbb{A}_{\mathrm{k}((t))}^{n}\right)$ is such that $g=\alpha^{-1} f \alpha$ has no pole at $t=0$, then $\alpha$ and $\alpha^{-1}$ have no pole at $t=0$. In particular, $g(0)$ is an element of $\operatorname{SAut}\left(\mathbb{A}_{\mathrm{k}}^{2}\right)$ that is conjugate to $f$. 
(2) For each $d \geq 1$, the set $\left\{g f g^{-1} \mid g \in \operatorname{SAut}\left(\mathbb{A}_{\mathrm{k}}^{n}\right)_{\leq d}\right\}$ is closed in $\operatorname{SAut}\left(\mathbb{A}_{\mathrm{k}}^{n}\right)$.

Proof. (1) We suppose that $\alpha$ has a pole at $t=0$, which is equivalent to the fact that $\alpha^{-1}$ has a pole at $t=0$ (Lemma 2.23), and show that $\alpha^{-1} f \alpha$ has a pole at $t=0$. If $X_{\alpha} \not \subset I_{f}$, this is given by Proposition 3.3. Otherwise, we have $X_{\alpha} \not \subset I_{f^{-1}}$, (because $I_{f} \cap I_{f^{-1}}=\varnothing$ by hypothesis) and apply the proposition to $f^{-1}$. This implies that $\alpha f^{-1} \alpha^{-1}$ has a pole at $t=0$. Hence, $\alpha f \alpha^{-1}$ has also a pole at $t=0$ by Lemma 2.23 .

(2) This follows from (1) and Corollary 2.26.

We obtain thus the following two results.

Proposition 3.5. Let $f \in \operatorname{SAut}\left(\mathbb{A}_{\mathrm{k}}^{n}\right)$ be a dynamically regular element having the following property: there exists a function $\tau: \mathbb{N} \rightarrow \mathbb{N}$ such that for each conjugate $g \in \operatorname{SAut}\left(\mathbb{A}_{\mathrm{k}}^{n}\right)$ of $f$, there exists $h \in \operatorname{SAut}\left(\mathbb{A}_{\mathrm{k}}^{n}\right)$ of degree $\leq \tau(\operatorname{deg}(g))$ such that $g=h f h^{-1}$.

Then, the conjugacy class of $f$ in $\operatorname{SAut}\left(\mathbb{A}_{\mathrm{k}}^{n}\right)$ is closed.

Proof. Let us denote by $C \subset \operatorname{SAut}\left(\mathbb{A}_{\mathrm{k}}^{n}\right)$ the conjugacy class of $f$ in $\operatorname{SAut}\left(\mathbb{A}_{\mathrm{k}}^{n}\right)$. Note that $C$ is closed (in $\operatorname{SAut}\left(\mathbb{A}_{\mathrm{k}}^{n}\right)$ ) if and only if

$$
C_{d}=\{g \in C \mid \operatorname{deg}(g) \leq d\}
$$

is closed in $\operatorname{SAut}\left(\mathbb{A}_{\mathrm{k}}^{n}\right)_{\leq d}$ for each $d \in \mathbb{N}$.

By Corollary 3.4, the set

$$
C_{d}^{\prime}=\left\{h f h^{-1} \mid h \in \operatorname{SAut}\left(\mathbb{A}_{\mathrm{k}}^{n}\right)_{\leq d}\right\}
$$

is closed in $\operatorname{SAut}\left(\mathbb{A}_{\mathrm{k}}^{n}\right)$ for each $d$.

By hypothesis, we have $C_{d} \subset C_{\tau(d)}^{\prime}$ for each $d \in \mathbb{N}$, which implies that

$$
C_{d}=C_{\tau(d)}^{\prime} \cap \operatorname{SAut}\left(\mathbb{A}_{\mathrm{k}}^{n}\right)_{\leq d}
$$

is closed in $\operatorname{SAut}\left(\mathbb{A}_{\mathrm{k}}^{n}\right)_{\leq d}$ for each $d$.

The additional hypothesis of Proposition 3.5 is fulfilled for all dynamically regular elements of $\operatorname{SAut}\left(\mathbb{A}_{\mathrm{k}}^{2}\right)$, so we obtain that the conjugacy classes of all dynamically regular elements of $\operatorname{SAut}\left(\mathbb{A}_{\mathrm{k}}^{2}\right)$ are closed:

Proposition 3.6. Let $f \in \operatorname{SAut}\left(\mathbb{A}_{\mathrm{k}}^{2}\right)$ be a dynamically regular element. Then:

(1) If $g \in \operatorname{SAut}\left(\mathbb{A}_{\mathrm{k}}^{2}\right)$ is conjugate to $f$, there exists $h \in \operatorname{SAut}\left(\mathbb{A}_{\mathrm{k}}^{2}\right)$, such that

$$
g=h f h^{-1} \quad \text { and } \quad \operatorname{deg}(h)^{2} \leq \operatorname{deg}(g) .
$$


(2) The conjugacy class of $f$ in $\operatorname{SAut}\left(\mathbb{A}_{\mathrm{k}}^{2}\right)$ is closed.

Remark 3.7. The bound of (1) also exists for $\operatorname{Bir}\left(\mathbb{A}_{\mathrm{k}}^{2}\right)$, but is much higher [Blanc and Cantat 2016].

Proof. Proposition 3.5 yields (1) $\Rightarrow$ (2), so we only need to prove (1).

Recall that, for each $g \in \operatorname{Aut}\left(\mathbb{A}_{\mathrm{k}}^{2}\right)$ of degree greater than one, $I_{g}=X_{g^{-1}}$ consists of one point (Remark 2.13).

Let $g=h f h^{-1}$ be an element of degree $d$. Replacing $h$ with $h f^{l}, l \in \mathbb{Z}$, we can assume that

$$
\operatorname{deg}(h) \leq \operatorname{deg}\left(h f^{l}\right), \quad \text { for each } l \in \mathbb{Z} .
$$

We can also assume that $\operatorname{deg}(h) \geq 2$. This implies, since $\operatorname{deg}(h)<\operatorname{deg}(h f) \operatorname{deg}\left(f^{-1}\right)$, that $I_{f}=X_{f^{-1}}=I_{h f}$ (Lemma 2.14). Similarly, we have $\operatorname{deg}(h)<\operatorname{deg}\left(h f^{-1}\right) \operatorname{deg}(f)$, so $I_{f^{-1}}=X_{f}=I_{h f^{-1}}$.

Because the two points $I_{f}, I_{f^{-1}} \in H_{\infty}$ are distinct, we have $I_{h} \neq I_{f}$ or $I_{h} \neq I_{f^{-1}}$. If $I_{h} \neq I_{f}=I_{h f}$, we have $\operatorname{deg}\left(h f h^{-1}\right)=\operatorname{deg}(h f) \operatorname{deg}\left(h^{-1}\right) \geq \operatorname{deg}(h) \operatorname{deg}\left(h^{-1}\right)$. If $I_{h} \neq$ $I_{f^{-1}}=I_{h f^{-1}}$, we have $\operatorname{deg}\left(h f^{-1} h^{-1}\right)=\operatorname{deg}\left(h f^{-1}\right) \operatorname{deg}\left(h^{-1}\right) \geq \operatorname{deg}(h) \operatorname{deg}\left(h^{-1}\right)$.

The degree of an element of $\operatorname{Aut}\left(\mathbb{A}_{\mathrm{k}}^{2}\right)$ and its inverse being the same, we find $\operatorname{deg}(g) \geq \operatorname{deg}(h)^{2}$.

Proof of Theorem 1.1. Part (1) and (2) correspond to the statements of Corollary 3.4. Part (3) is provided by Proposition 3.6.

It remains to show (4). For each $d \in \mathbb{N}$, the set

$$
C_{d}=\left\{g f g^{-1} \mid g \in \operatorname{SAut}\left(\mathbb{A}_{\mathrm{k}}^{n}\right)_{\leq d}\right\}
$$

is closed in $\operatorname{SAut}\left(\mathbb{A}_{\mathrm{k}}^{n}\right)$, and the conjugacy class of $f$ is the infinite union $C=\bigcup_{d} C_{d}$. Let $A$ be an algebraic variety, $F: A \rightarrow \operatorname{SAut}\left(\mathbb{A}_{\mathrm{k}}^{n}\right)$ be a morphism, and let $B \subset A$ be a locally closed subset which is contained in $F^{-1}(C)$. Writing $B_{d}=F^{-1}\left(C_{d}\right)$, the set $B_{d}$ is closed in $B$ for each $d$ and $B=\bigcup_{d} B_{d}$. Since $\mathrm{k}$ is uncountable and $B_{d} \subset B_{d+1}$ for each $d$, we obtain $B=B_{m}$ for some integer $m$. Hence, $B$ is contained in $F^{-1}\left(C_{m}\right)$, which is closed in $A$, so the closure of $B$ in $A$ is also contained in $F^{-1}\left(C_{m}\right)$, and thus in $F^{-1}(C)$.

\section{Conjugacy classes of elements in $\operatorname{SAut}\left(\mathbb{A}_{K}^{2}\right)$}

4A. Overview of the Jung-van der Kulk theorem and its applications. For each field $K$, the Jung-van der Kulk theorem [Jung 1942; van der Kulk 1953] asserts that the group $\operatorname{Aut}\left(\mathbb{A}_{K}^{2}\right)$ is generated by the groups

$$
\begin{aligned}
\operatorname{Aff}\left(\mathbb{A}_{K}^{2}\right) & =\left\{\left(a x_{1}+b x_{2}+e, c x_{1}+d x_{2}+f\right) \mid\left(\begin{array}{ll}
a & b \\
c & d
\end{array}\right) \in \mathrm{GL}(2, K), e, f \in K\right\}, \\
\mathrm{J}\left(\mathbb{A}_{K}^{2}\right) & =\left\{\left(a x_{1}+P\left(x_{2}\right), b x_{2}+c\right) \mid a, b \in K^{*}, c \in K, P \in K\left[x_{2}\right]\right\} .
\end{aligned}
$$


Multiplying the decomposition of an element of $\operatorname{SAut}\left(\mathbb{A}_{K}^{2}\right)$ with homotheties we can assume that each one has determinant 1. This implies that the group $\operatorname{SAut}\left(\mathbb{A}_{K}^{2}\right)$ is generated by the groups

$$
\begin{aligned}
\operatorname{SAff}\left(\mathbb{A}_{K}^{2}\right) & =\left\{\left(a x_{1}+b x_{2}+e, c x_{1}+d x_{2}+f\right) \mid\left(\begin{array}{ll}
a & b \\
c & d
\end{array}\right) \in \operatorname{SL}(2, K), e, f \in K\right\}, \\
\operatorname{SJ}\left(\mathbb{A}_{K}^{2}\right) & =\left\{\left(a x_{1}+P\left(x_{2}\right), a^{-1} x_{2}+c\right) \mid a \in K^{*}, c \in K, P \in K\left[x_{2}\right]\right\} .
\end{aligned}
$$

The Jung-van der Kulk theorem implies that we have an amalgamated product structure on $\operatorname{SAut}\left(\mathbb{A}_{K}^{2}\right)$. It also yields the following classical result. We recall here the simple proof.

Lemma 4.1. Every element $f \in \operatorname{SAut}\left(\mathbb{A}_{K}^{2}\right)$ is conjugate either to an element of $\mathrm{SJ}\left(\mathbb{A}_{K}^{2}\right)$ or to an element of the form

$$
f=a_{m} j_{m} \cdots a_{1} j_{1},
$$

where $m \geq 1$ and each $a_{i} \in \operatorname{SAff}\left(\mathbb{A}_{K}^{2}\right) \backslash \operatorname{SJ}\left(\mathbb{A}_{K}^{2}\right)$ and each $j_{i} \in \operatorname{SJ}\left(\mathbb{A}_{K}^{2}\right) \backslash \operatorname{SAff}\left(\mathbb{A}_{K}^{2}\right)$. Proof. We write $f$ as a product of elements of $A=\operatorname{SAff}\left(\mathbb{A}_{K}^{2}\right)$ and $J=\operatorname{SJ}\left(\mathbb{A}_{K}^{2}\right)$ :

$$
f=a_{m} j_{m} a_{m-1} \cdots a_{1} j_{1} a_{0},
$$

where each $a_{i} \in A$ and each $j_{i} \in J$. By merging elements we can moreover assume that $j_{i} \notin A$ for $i=1, \ldots, m$, and that $a_{i} \notin J$ for $i=1, \ldots, m-1$.

Suppose first that $m=0$, which implies that $f=a_{0} \in A$, so it extends to an element of $\operatorname{Aut}\left(\mathbb{P}_{K}^{2}\right)$. The action at infinity has a fixed point, and conjugating by an element of $\operatorname{SL}(2, K)$, we can assume that this point corresponds to $[0: 1: 0]$, which implies that $f$ preserves the pencil of lines through the point, so $f \in J$.

Suppose now that $m \geq 1$, which implies that $j_{1} \in J \backslash A$. We conjugate $f$ by $a_{0}$ and assume that $a_{0}=$ id. If $m=1$ and $a_{m}=a_{1} \in J$, then $f \in J$. If $m \geq 2$ and $a_{m} \in J$, we conjugate by $j_{1}$ and decrease $m$ by 1 . This reduces to the case $m \geq 1$, $a_{1}=\mathrm{id}, a_{m} \notin J$.

Remark 4.2. Elements of the form $f=a_{m} j_{m} \cdots a_{1} j_{1}$, where $m \geq 1$ and each $a_{i} \in \operatorname{SAff}\left(\mathbb{A}_{K}^{2}\right) \backslash \operatorname{SJ}\left(\mathbb{A}_{K}^{2}\right)$ and each $j_{i} \in \operatorname{SJ}\left(\mathbb{A}_{K}^{2}\right) \backslash \operatorname{SAff}\left(\mathbb{A}_{K}^{2}\right)$ are usually called Hénon automorphisms. Note that each element $j_{i}$ satisfies $X_{j_{i}}=I_{j_{i}}=[0: 1: 0]$, and that each $a_{i}$ extends to an automorphism of $\mathbb{P}_{K}^{2}$ that moves [0:1:0]. By Lemma 2.14, this implies that

$$
\operatorname{deg}(f)=\prod_{i=1}^{m} \operatorname{deg}\left(j_{i}\right), \quad I_{f}=[0: 1: 0], \quad X_{f}=I_{f^{-1}}=a_{m}([0: 1: 0]) .
$$

In particular, $I_{f} \cap I_{f^{-1}}=\varnothing, \operatorname{deg}(f)>1$ and $\operatorname{deg}\left(f^{m}\right)=\operatorname{deg}(f)^{m}$ for each $m \geq 1$ (Corollary 2.17). Moreover, the conjugacy class of $f$ in $\operatorname{SAut}\left(\mathbb{A}_{\bar{K}}^{2}\right)$ is closed, where $\bar{K}$ is the algebraic closure of $K$ (Proposition 3.6). 
Remark 4.3. Elements of $\operatorname{SJ}\left(\mathbb{A}_{K}^{2}\right)$ are algebraic since they are contained in an algebraic subgroup of $\operatorname{Aut}\left(\mathbb{A}_{K}^{2}\right)$ (or equivalently have a bounded degree sequence). Hénon automorphisms are not algebraic as their degree sequence is not bounded. Hence, Lemma 4.1 corresponds to saying that nonalgebraic elements are conjugate to Hénon automorphisms, and algebraic automorphisms to elements of $\operatorname{SJ}\left(\mathbb{A}_{K}^{2}\right)$.

The following corollary follows from Lemma 4.1, and also holds (with the same proof) for $\operatorname{Aut}\left(\mathbb{A}_{\mathrm{k}}^{2}\right)$. This was already observed in [Furter 1999].

Corollary 4.4. (1) An element $f \in \operatorname{SAut}\left(\mathbb{A}_{\mathrm{k}}^{2}\right)$ is algebraic if and only if

$$
\operatorname{deg}\left(f^{2}\right) \leq \operatorname{deg}(f) .
$$

(2) The subset $\operatorname{SAut}\left(\mathbb{A}_{\mathrm{k}}^{2}\right)_{\text {alg }}$ of algebraic elements of $\operatorname{SAut}\left(\mathbb{A}_{\mathrm{k}}^{2}\right)$ is closed.

Proof. (1)(a) If $f$ is algebraic it is conjugate to an element $j \in \operatorname{SJ}\left(\mathbb{A}_{K}^{2}\right)($ Lemma 4.1, or more precisely Remark 4.3). We can thus write $f=\alpha^{-1} j \alpha$ where $\alpha \in \operatorname{SAut}\left(\mathbb{A}_{K}^{2}\right)$. Then $\alpha=a_{1} j_{1} \cdots$, where each $a_{i} \in \operatorname{SAff}\left(\mathbb{A}_{K}^{2}\right) \backslash \operatorname{SJ}\left(\mathbb{A}_{K}^{2}\right)$ and each $j_{i} \in \operatorname{SJ}\left(\mathbb{A}_{K}^{2}\right)$ (if $\alpha$ starts with an element of $\operatorname{SJ}\left(\mathbb{A}_{K}^{2}\right) \backslash \operatorname{SAff}\left(\mathbb{A}_{K}^{2}\right)$ we can merge this element with $\left.j\right)$. If $j \notin \operatorname{SAff}\left(\mathbb{A}_{K}^{2}\right)$, then $\operatorname{deg}(f)=\prod \operatorname{deg}\left(j_{i}\right)^{2} \operatorname{deg}(j)=\operatorname{deg}\left(\alpha^{-1}\right) \operatorname{deg}(j) \operatorname{deg}(\alpha)$ (see Remark 4.2) and thus

$$
\operatorname{deg}\left(f^{2}\right)=\operatorname{deg}\left(\alpha^{-1} j^{2} \alpha\right) \leq \operatorname{deg}\left(\alpha^{-1}\right) \operatorname{deg}\left(j^{2}\right) \operatorname{deg}(\alpha) \leq \operatorname{deg}(f),
$$

since $\operatorname{deg}\left(j^{2}\right) \leq \operatorname{deg}(j)$. If $j \in \operatorname{SAff}\left(\mathbb{A}_{K}^{2}\right) \cap \operatorname{SJ}\left(\mathbb{A}_{K}^{2}\right)$, then we can replace $j$ with $a_{1}^{-1} j a_{1}$ and continue like this to obtain either the previous case or $f=\alpha^{-1} a \alpha$, where $a \in \operatorname{SAff}\left(\mathbb{A}_{K}^{2}\right), \alpha=j_{1} a_{1} \cdots$, each $a_{i} \in \operatorname{SAff}\left(\mathbb{A}_{K}^{2}\right) \backslash \operatorname{SJ}\left(\mathbb{A}_{K}^{2}\right)$ and each $j_{i} \in$ $\operatorname{SJ}\left(\mathbb{A}_{K}^{2}\right) \backslash \operatorname{SAff}\left(\mathbb{A}_{K}^{2}\right)$. We obtain similarly $\operatorname{deg}\left(f^{2}\right) \leq \operatorname{deg}(f)$.

(1)(b) If $f$ is not algebraic, then $f$ is conjugate to a Hénon map $h=a_{m} j_{m} \cdots a_{1} j_{1}$, where each $a_{i} \in \operatorname{SAff}\left(\mathbb{A}_{K}^{2}\right) \backslash \operatorname{SJ}\left(\mathbb{A}_{K}^{2}\right)$ and each $j_{i} \in \operatorname{SJ}\left(\mathbb{A}_{K}^{2}\right) \backslash \operatorname{SAff}\left(\mathbb{A}_{K}^{2}\right)(\operatorname{Lemma} 4.1$, or more precisely Remark 4.3). Writing $f=\alpha^{-1} h \alpha$, where $\alpha \in \operatorname{SAut}\left(\mathbb{A}_{K}^{2}\right)$, we can write as before $\alpha$ as a product of elements of $\operatorname{SAff}\left(\mathbb{A}_{K}^{2}\right)$ and $\operatorname{SJ}\left(\mathbb{A}_{K}^{2}\right)$, replace $h$ if $\alpha$ starts with an element $j_{1}^{\prime} \in \operatorname{SJ}\left(\mathbb{A}_{K}^{2}\right)$ such that $j_{1} j_{1}^{\prime} \in \operatorname{SAff}\left(\mathbb{A}_{K}^{2}\right)$ or if it starts with an element $a_{1}^{\prime} \in \operatorname{SAff}\left(\mathbb{A}_{K}^{2}\right)$ such that $\left(a_{1}^{\prime}\right)^{-1} \alpha_{m} \in \operatorname{SJ}\left(\mathbb{A}_{K}^{2}\right)$ and finish with a simplified writing where we can directly read that $\operatorname{deg}\left(f^{2}\right)>\operatorname{deg}(f)$.

(2) It follows from (1) that the set of algebraic elements of $\operatorname{SAut}\left(\mathbb{A}_{K}^{2}\right)_{\leq d}$ is closed for each $d \geq 1$, which implies the second assertion.

4B. Conjugacy classes of elements of $\mathbf{S J}\left(\mathbb{A}_{K}^{2}\right)$. It is easy to decide whether two Hénon automorphisms are conjugate, using the amalgamated product structure. The writing of such an element is unique up to cycling permutation of the elements and up to inserting elements of $\operatorname{SJ}\left(\mathbb{A}_{K}^{2}\right) \cap \operatorname{SAff}\left(\mathbb{A}_{K}^{2}\right)$. The classification of conjugacy classes of $\operatorname{SAut}\left(\mathbb{A}_{K}^{2}\right)$ thus reduces to the study of elements of $\operatorname{SJ}\left(\mathbb{A}_{K}^{2}\right)$. 
Lemma 4.5. Let $f \in \mathrm{SJ}\left(\mathbb{A}_{K}^{2}\right)$ be some element. After conjugation in this group, the element belongs to one of the following families:

(i) $\left(a x_{1}, a^{-1} x_{2}\right)$,

$a \in K \backslash\{0,1\}$,

(ii) $\left(x_{1}+P\left(x_{2}\right), x_{2}\right)$, $P \in K\left[x_{2}\right]$,

(iii) $\left(\zeta x_{1}+x_{2}^{m-1} P\left(x_{2}^{m}\right), \zeta^{-1} x_{2}\right)$,

$\zeta \in K \backslash\{0,1\}, \quad P \in K\left[x_{2}\right] \backslash\{0\}$, $\zeta$ a primitive $m$-th root of unity,

$$
\left\{\begin{array}{l}
\left(x_{1}, x_{2}+1\right) \\
\left(x_{1}+x_{2}^{p-1} P\left(x_{2}^{p}\right), x_{2}+1\right)
\end{array}\right.
$$
$\operatorname{char}(K)=0$, $P \in K\left[x_{2}\right], \quad \operatorname{char}(K)=p$.

Proof. An element $f \in \mathrm{SJ}\left(\mathbb{A}_{K}^{2}\right)$ is equal to $\left(a x_{1}+P\left(x_{2}\right), a^{-1} x_{2}+c\right)$ for some $a \in K^{*}, c \in K, P \in K\left[x_{2}\right]$.

If $a \neq 1$, we conjugate by $\left(x_{1}, x_{2}+a c /(1-a)\right)$ and can assume that $c=0$. Conjugating by $\left(x_{1}-\lambda x_{2}^{n}, x_{2}\right)$ yields $\left(a x_{1}+P\left(x_{2}\right)+\lambda\left(a-a^{-n}\right) x_{2}^{n}, a^{-1} x_{2}\right)$. We can then kill the coefficient of degree $n$ of $P$, if $a \neq a^{-n}$. This gives (i) or (iii).

If $a=1$ and $c=0$, we get (ii). If $a=1$ and $c \neq 0$, we conjugate by $\left(c x_{1}, c^{-1} x_{2}\right)$ and can assume that $c=1$. Conjugating $\left(x_{1}+P\left(x_{2}\right), x_{2}+1\right)$ by $\left(x_{1}-\lambda x_{2}^{n+1}, x_{2}\right)$ yields $\left(x_{1}+P\left(x_{2}\right)+\lambda\left(x_{2}^{n+1}-\left(x_{2}+1\right)^{n+1}\right), x_{2}+1\right)$. We can thus kill the coefficient of degree $n$ if $n+1$ is not a multiple of $\operatorname{char}(K)$. This gives (iv).

The most complicated case corresponds to family (iv), in the case of a field of characteristic $p>0$. In order to describe the conjugacy classes in this family, we will need the following lemma.

Lemma 4.6. Let $K$ be a field of characteristic $p>0$. Let $V \subset K[x]$ be the subvector space given by

$$
V=\left\{x^{p-1} P\left(x^{p}\right) \mid P \in K[x]\right\},
$$

and let $\delta, N$ be the following $K$-linear maps

$$
\begin{aligned}
\delta: K[x] \longrightarrow K[x], & F(x) \longmapsto F(x+1)-F(x), \\
N: K[x] \longrightarrow K[x], \quad F(x) & \longmapsto F(x)+F(x+1)+\cdots+F(x+p-1) .
\end{aligned}
$$

Then, $\operatorname{Im}(\delta)=\operatorname{Ker}(N)$ and $K[x]=V \oplus \operatorname{Im}(\delta)=V \oplus \operatorname{Ker}(N)$.

Proof. (1) We first show that $V+\operatorname{Im}(\delta)=K[x]$. This is the same argument as in Lemma 4.5(iv). We take any polynomial $P(x)=\sum_{j=1}^{l} a_{j} x^{j} \in K[x]$. If $P \notin V$, we can define $m$ to be the biggest integer such that $a_{m} \neq 0$ and $m+1 \neq \equiv(\bmod p)$. Replacing $P$ with $\tilde{P}(x)=P(x)-\delta\left(a_{m} x^{m+1} /(m+1)\right)$, we kill the coefficient of $x^{m}$. Continuing this process until all coefficients $a_{i}$ with $i+1 \not \equiv 0(\bmod p)$ are zero, we obtain an element of $V$. 
(2) We now show that $\operatorname{Ker}(N) \cap V=\{0\}$. Let $F(x)=\sum_{i=1}^{n} a_{i} x^{i p-1}$ be an element of $V$, and assume that $a_{n} \neq 0$. Since

$$
N(F(x))=\sum_{i=1}^{n} a_{i}\left(\sum_{k=0}^{p-1}(x+k)^{i p-1}\right),
$$

the coefficient of $x^{n p-p}$ of $N(F(x))$ is equal to

$$
a_{n}\left(\begin{array}{c}
n p-1 \\
n p-p
\end{array}\right) \sum_{k=0}^{p-1} k^{p-1}=a_{n}\left(\begin{array}{c}
n p-1 \\
n p-p
\end{array}\right)(p-1) \in K^{*} .
$$

This shows that $F \notin \operatorname{Ker}(N)$. Hence $V \cap \operatorname{Ker}(N)=\{0\}$.

(3) The inclusion $\operatorname{Im}(\delta) \subset \operatorname{Ker}(N)$ follows from the definition of $\delta$ and $N$, so we obtain $V \cap \operatorname{Im}(\delta)=\{0\}$ by (2). Moreover, since $\operatorname{Ker}(N)$ is contained in $\operatorname{Im}(\delta) \oplus V$ (by (1)) and $\operatorname{Ker}(N) \cap V=\{0\}$, we have $\operatorname{Im}(\delta)=\operatorname{Ker}(N)$.

Remark 4.7. The equality $\operatorname{Ker}(N) \cap V=\{0\}$ of Lemma 4.6 corresponds to the fact that an element of the form

$$
\left(x_{1}+x_{2}^{p-1} P\left(x_{2}^{p}\right), x_{2}+1\right),
$$

where $P \in K\left[x_{2}\right], \operatorname{char}(K)=p$ (family (iv)) is of order $p$ if and only if $P=0$. Indeed,

$$
\left(x_{1}+Q\left(x_{2}\right), x_{2}+1\right)^{p}=\left(x_{1}+Q\left(x_{2}\right)+\cdots+Q\left(x_{2}+p-1\right), x_{2}\right),
$$

for each $Q \in K\left[x_{2}\right]$.

We will also need the following lemma, which is a direct consequence of the amalgamated product structure of $\operatorname{SAut}\left(\mathbb{A}_{K}^{2}\right)$.

Lemma 4.8. Let $f \in \mathrm{SJ}\left(\mathbb{A}_{K}^{2}\right)$, which is not conjugate to $\left(a x_{1}, a^{-1} x_{2}\right), a \in K^{*}$ or to $\left(x_{1}+1, x_{2}\right)$ or $\left(x_{1}, x_{2}+1\right)$ in the group $\mathrm{SJ}\left(\mathbb{A}_{K}^{2}\right)$. Then, $f$ is conjugate to an element of $g \in \mathrm{SJ}\left(\mathbb{A}_{K}^{2}\right)$ in the group $\operatorname{SAut}\left(\mathbb{A}_{K}^{2}\right)$ if and only if it is conjugate to $g$ in $\mathrm{SJ}\left(\mathbb{A}_{K}^{2}\right)$.

Proof. We suppose that $g=\alpha f \alpha^{-1} \in \operatorname{SJ}\left(\mathbb{A}_{K}^{2}\right)$, for some $\alpha \in \operatorname{SAut}\left(\mathbb{A}_{K}^{2}\right) \backslash \operatorname{SJ}\left(\mathbb{A}_{K}^{2}\right)$. We can write $\alpha$ as

$$
\alpha=j_{m+1} a_{m} j_{m} \cdots j_{2} a_{1} j_{1},
$$

where $m \geq 1$, each $j_{i} \in \operatorname{SJ}\left(\mathbb{A}_{K}^{2}\right)$, each $a_{i} \in \operatorname{SAff}\left(\mathbb{A}_{K}^{2}\right)$, and such that $a_{i} \notin \operatorname{SJ}\left(\mathbb{A}_{K}^{2}\right)$ for $i=1, \ldots, m, j_{i} \notin \operatorname{SAff}\left(\mathbb{A}_{K}^{2}\right)$ for $i=2, \ldots, m-1$. Since $\alpha f \alpha^{-1} \in \operatorname{SJ}\left(\mathbb{A}_{K}^{2}\right)$, the element $j_{1} f j_{1}^{-1}$ belongs to $\operatorname{SAff}\left(\mathbb{A}_{K}^{2}\right) \cap \operatorname{SJ}\left(\mathbb{A}_{K}^{2}\right)$, and the same holds for the element $a_{1} j_{1} f j_{1}^{-1} a_{1}^{-1}$.

The fact that $f_{1}=j_{1} f j_{1}^{-1}$ and $f_{2}=a_{1} j_{1} f j_{1}^{-1} a_{1}^{-1}$ belong to $\operatorname{SAff}\left(\mathbb{A}_{K}^{2}\right) \cap \operatorname{SJ}\left(\mathbb{A}_{K}^{2}\right)$ corresponds to the fact that both extend to automorphisms of $\mathbb{P}_{K}^{2}$ that fix the point $[0: 1: 0]$ at infinity. However, $a_{1} \in \operatorname{SAff}\left(\mathbb{A}_{K}^{2}\right) \backslash \operatorname{SJ}\left(\mathbb{A}_{K}^{2}\right)$ so it extends to an 
automorphism of $\mathbb{P}_{K}^{2}$, whose action at the infinity moves the point $[0: 1: 0]$. This implies that $f_{1}$ fixes at least two points at infinity. Conjugating by an element of $\operatorname{SAff}\left(\mathbb{A}_{K}^{2}\right) \cap \operatorname{SJ}\left(\mathbb{A}_{K}^{2}\right)$ we can assume that these points are $[0: 1: 0]$ and $[0: 0: 1]$. This implies that $f_{1}=\left(a x_{1}+b, a^{-1} x_{2}+c\right)$, for some $(a, b, c) \in K^{*} \times K^{2}$. It remains to see that $f_{1}$ is conjugate to $\left(a x_{1}, a^{-1} x_{2}\right),\left(x_{1}+1, x_{2}\right)$ or $\left(x_{1}, x_{2}+1\right)$, in the group $\operatorname{SJ}\left(\mathbb{A}_{K}^{2}\right)$. This is a straight-forward calculation, already done in the proof of Lemma 4.5.

Proposition 4.9 (Conjugacy classes of algebraic elements in $\operatorname{SAut}\left(\mathbb{A}_{K}^{2}\right)$ ). Every algebraic element of $\operatorname{SAut}\left(\mathbb{A}_{K}^{2}\right)$ is conjugate to one of the families (i)-(iv) of Lemma 4.5.

Apart from $\left(x_{1}, x_{2}+1\right)$, which is conjugate to $\left(x_{1}+1, x_{2}\right)$, no two elements of distinct families are conjugate in $\operatorname{SAut}\left(\mathbb{A}_{K}^{2}\right)$. Moreover, the conjugacy classes in $\operatorname{SAut}\left(\mathbb{A}_{K}^{2}\right)$ within the families are as follows:

(i) $\left(a x_{1}, a^{-1} x_{1}\right) \sim\left(b x_{1}, b^{-1} x_{2}\right) \Longleftrightarrow a=b^{ \pm 1}$.

(ii) $\left(x_{1}+P\left(x_{2}\right), x_{2}\right) \sim\left(x_{1}+Q\left(x_{2}\right), x_{2}\right) \Longleftrightarrow Q\left(x_{2}\right)=a P\left(a x_{2}+b\right)$ for some $(a, b) \in K^{*} \times K$.

(iii) $\left(\zeta x_{1}+x_{2}^{m-1} P\left(x_{2}^{m}\right), \zeta^{-1} x_{2}\right)$ is conjugate to $\left(\zeta x_{1}+x_{2}^{m-1} a^{m} P\left(a x_{2}^{m}\right), \zeta^{-1} x_{2}\right)$ for each $a \in K^{*}$, but not to any other element of family (iii).

(iv) Two elements

$$
f=\left(x_{1}+x_{2}^{p-1} P\left(x_{2}^{p}\right), x_{2}+1\right), \quad g=\left(x_{1}+x_{2}^{p-1} Q\left(x_{2}^{p}\right), x_{2}+1\right),
$$

where $P, Q \in K\left[x_{2}\right]$, are conjugate if and only their $p$-th powers,

$$
f^{p}=\left(x_{1}+\tilde{P}\left(x_{2}\right), x_{2}\right), \quad g^{p}=\left(x_{1}+\tilde{Q}\left(x_{2}\right), x_{2}\right),
$$

where $\tilde{P}, \tilde{Q} \in K\left[x_{2}\right]$, satisfy $\tilde{Q}\left(x_{2}\right)=\tilde{P}\left(x_{2}+c\right)$ for some $c \in K$.

Proof. The first assertion follows from Lemma 4.1 (see Remark 4.3). It remains to describe the conjugacy classes in the families (i)-(iv). We first observe that if $\alpha \in \operatorname{SL}(2, K)$ is conjugate by $v \in \operatorname{SAut}\left(\mathbb{A}_{K}^{2}\right)$ to an element $\beta \in \operatorname{SAut}\left(\mathbb{A}_{K}^{2}\right)$ that fixes the origin, the derivative of the equation $\alpha v=v \beta$ at the origin yields $\alpha D_{v}(0)=D_{v}(0) D_{\beta}(0)$, so the derivative of $\beta$ at the origin is conjugate to $\alpha$ in $\mathrm{SL}(2, K)$. This shows that the families (i) and (ii) are disjoint, and gives the conjugacy classes in (i).

We now observe that two elements $f=\left(x_{1}+P\left(x_{2}\right), x_{2}\right)$ and $\left(x_{1}+Q\left(x_{2}\right), x_{2}\right)$ are conjugate in $\operatorname{SAut}\left(\mathbb{A}_{K}^{2}\right)$ if and only if they are conjugate in $\operatorname{SJ}\left(\mathbb{A}_{K}^{2}\right)$. This is given by Lemma 4.8 , if $f$ or $g$ is not conjugate to $\left(x_{1}+1, x_{2}\right)$ or $\left(x_{1}, x_{2}\right)$ in $\operatorname{SJ}\left(\mathbb{A}_{K}^{2}\right)$, and is trivial in the remaining cases (when both $f$ and $g$ are conjugate to one of these two elements). It remains to observe that the conjugation of $f=\left(x_{1}+P\left(x_{2}\right), x_{2}\right)$ by 
$\left(a x_{1}+R\left(x_{2}\right), a^{-1}\left(x_{2}-b\right)\right)$ yields $\left(x_{1}+a P\left(a x_{2}+b\right), x_{2}\right)$ to obtain the conjugacy classes in (ii).

An element $f=\left(\zeta x_{1}+x_{2}^{m-1} P\left(x_{2}^{m}\right), \zeta^{-1} x_{2}\right)$ in family (iii) satisfies

$$
f^{m}=\left(x_{1}+m x_{2}^{m-1} P\left(x_{2}^{m}\right), x_{2}\right) \neq\left(x_{1}, x_{2}\right) .
$$

Since $f^{m}$ belongs to family (ii), $f^{m}$ (and thus $f$ ) is not conjugate to an element of family (i). Moreover, $f$ is not conjugate to an element of family (ii) because $\zeta^{-1} \in K \backslash\{0,1\}$ is an eigenvalue of the action of $f^{*}$ on $K\left[x_{1}, x_{2}\right]$. By Lemma 4.8, $f$ is conjugate to another element $g$ of family (iii) in $\operatorname{SAut}\left(\mathbb{A}_{K}^{2}\right)$ if and only if this conjugation holds in $\operatorname{SJ}\left(\mathbb{A}_{K}^{2}\right)$. Looking at the second coordinate, we have to conjugate by $\alpha=\left(a x_{1}+R\left(x_{2}\right), a^{-1} x_{2}\right)$. This implies that $g=\left(\zeta x_{1}+x_{2}^{m-1} Q\left(x_{2}^{m}\right), \zeta^{-1} x_{2}\right)$ for some polynomial $Q$. Looking at $g^{m}=\alpha f^{m} \alpha^{-1}=\left(x_{1}+m a^{m} x_{2}^{m-1} P\left(\left(a x_{2}\right)^{m}\right), x_{2}\right)$, we obtain $Q\left(x_{2}^{m}\right)=a^{m} P\left(\left(a x_{2}\right)^{m}\right)$. This yields a necessary condition on $Q$, which is also sufficient, by choosing $R=0$.

It remains to study the last class (iv). The element $\left(x_{1}, x_{2}+1\right)$ is conjugate to $\left(x_{1}+1, x_{2}\right)$ by $\left(x_{2},-x_{1}\right)$. If $\operatorname{char}(K)=0$, there is no other element in the family. So we assume that $\operatorname{char}(K)=p>0$, and consider $f=\left(x_{1}+x_{2}^{p-1} P\left(x_{2}^{p}\right), x_{2}+1\right)$, for some polynomial $P \in K\left[x_{2}\right] \backslash\{0\}$. Because the action of $f^{*}$ on $K\left[x_{1}, x_{2}\right]$ has only eigenvalues equal to $1, f$ is not conjugate to any element of family (i) or (iii). Moreover, the fact that $P \neq 0$ implies that $f^{p}$ is not the identity (see Remark 4.7). Hence, $f$ is not conjugate to an element of family (ii).

We then take $g=\left(x_{1}+x_{2}^{p-1} Q\left(x_{2}^{p}\right), x_{2}+1\right)$, for some $Q \in K\left[x_{2}\right]$, write

$$
f^{p}=\left(x_{1}+\tilde{P}\left(x_{2}\right), x_{2}\right), \quad g^{p}=\left(x_{1}+\tilde{Q}\left(x_{2}\right), x_{2}\right),
$$

for some $\tilde{P}, \tilde{Q} \in K\left[x_{2}\right]$, and show that $f$ and $g$ are conjugate if and only if $\tilde{Q}\left(x_{2}\right)=\tilde{P}\left(x_{2}+c\right)$ for some $c \in K$. This will conclude the proof.

Suppose first that $f$ is conjugate to $g$. Lemma 4.8 yields the existence of $\alpha \in \operatorname{SJ}\left(\mathbb{A}_{K}^{2}\right)$ such that $\alpha f \alpha^{-1}=g$. Looking at the second coordinate, we see that $\alpha=\left(x_{1}+R\left(x_{2}\right), x_{2}-c\right)$ for some $R \in K\left[x_{2}\right], c \in K$. Then, $\alpha f^{p} \alpha^{-1}=$ $\left(x_{1}+\tilde{P}\left(x_{2}+c\right), x_{2}\right)$, which implies that $\tilde{Q}\left(x_{2}\right)=\tilde{P}\left(x_{2}+c\right)$ as we wanted.

Conversely, suppose that $\tilde{Q}\left(x_{2}\right)=\tilde{P}\left(x_{2}+c\right)$ for some $c \in K$. We conjugate then $g$ with $\left(x_{1}, x_{2}+c\right)$; this changes maybe the polynomial $Q$, and replaces $\tilde{Q}$ with $\tilde{P}$. Hence, we can assume that $f^{p}=g^{p}$. This corresponds to the equality

$$
N\left(x_{2}^{p-1} P\left(x_{2}^{p}\right)\right)=N\left(x_{2}^{p-1} Q\left(x_{2}^{p}\right)\right),
$$

where $N: K\left[x_{2}\right] \rightarrow K\left[x_{2}\right]$ is the $K$-linear map that sends $f\left(x_{2}\right)$ to $f\left(x_{2}\right)+$ $f\left(x_{2}+1\right)+\cdots+f\left(x_{2}+p-1\right)$. The element $x_{2}^{p-1} Q\left(x_{2}^{p}\right)-x_{2}^{p-1} P\left(x_{2}^{p}\right)$ is an element of the kernel of $N$, and is thus equal to $R\left(x_{2}+1\right)-R\left(x_{2}\right)$ for some polynomial $R \in K\left[x_{2}\right]$ (Lemma 4.6). This corresponds to the fact that $f$ is conjugate to $g$ by $\alpha=\left(x_{1}+R\left(x_{2}\right), x_{2}\right)$. 
4C. Degenerations between the four families of conjugacy classes. In the remaining part of the article, we show that every algebraic element of $\operatorname{SAut}\left(\mathbb{A}_{K}^{2}\right)$ admits a degeneration of conjugates towards a diagonalisable element.

4C1. From family (ii) and (iii) to family (i). These are the easiest degenerations. Taking any $f \in \operatorname{SAut}\left(\mathbb{A}_{K}^{2}\right)$ that belongs to family (ii) or (iii), and conjugating by diagonal elements of $\operatorname{SAut}\left(\mathbb{A}_{K(t)}^{2}\right)$ we obtain elements of $\operatorname{SAut}\left(\mathbb{A}_{K[t]}^{2}\right)$ which yield families of conjugates of $f$ that converge towards an element of family (i). Indeed, we have

$$
\begin{aligned}
\left(t x_{1}, t^{-1} x_{2}\right)\left(x_{1}+P\left(x_{2}\right), x_{2}\right)\left(t^{-1} x_{1}, t x_{2}\right) & =\left(x_{1}+t P\left(x_{2}\right), x_{2}\right), \\
\left(t x_{1}, t^{-1} x_{2}\right) f\left(t^{-1} x_{1}, t x_{2}\right) & =\left(\zeta x_{1}+t^{m} x_{2}^{m-1} P\left(t x_{2}^{m}\right), \zeta^{-1} x_{2}\right) .
\end{aligned}
$$

In particular, there is a diagonalisable element in the closure of the conjugacy class of any element of families (ii) and (iii), and thus of any algebraic element of $\operatorname{SAut}\left(\mathbb{A}_{K}^{2}\right)$, if $\operatorname{char}(K)=0$.

4C2. Family (iv). The last family, when $\operatorname{char}(K)=p>0$, is the most interesting. The elements of this family are of the form $\left(x_{1}+Q\left(x_{2}\right), x_{2}+1\right)$, for some polynomial $Q \in K\left[x_{2}\right]$. Diagonal conjugations by $\left(t x_{1}, t^{-1} x_{2}\right)$ or $\left(t^{-1} x_{1}, t x_{2}\right)$ yield elements of $\operatorname{SAut}\left(\mathbb{A}_{K\left[t^{ \pm 1}\right]}^{2}\right)$ which do not have a value at $t=0$.

In the following proposition, we provide two explicit degenerations, typical to positive characteristic, to either $\left(x_{1}, x_{2}+1\right)$ or the identity.

Proposition 4.10. Assume that $\operatorname{char}(K)=p>0$ and let

$$
f=\left(x_{1}+Q\left(x_{2}\right), x_{2}+1\right)
$$

for some polynomial $Q \in K\left[x_{2}\right]$.

Then, there are two elements $F_{1}, F_{2} \in \operatorname{SAut}\left(\mathbb{A}_{K[t]}^{2}\right)$, which are conjugate to $f$ in $\operatorname{SAut}\left(\mathbb{A}_{K\left[t^{ \pm 1}\right]}^{2}\right)$, and have the following properties:

(1) For each $t \in K \backslash\{0\}, F_{1}(t), F_{2}(t) \in \operatorname{SAut}\left(\mathbb{A}_{K}^{2}\right)$ are conjugate to $f$ in $\operatorname{SAut}\left(\mathbb{A}_{K}^{2}\right)$.

(2) $F_{1}(0)=\left(x_{1}, x_{2}+1\right), \quad F_{2}(0)=\left(x_{1}, x_{2}\right)$.

Proof. If $Q=0$, we can choose $F_{1}(t)=f$ and $F_{2}(t)$ to be the conjugation of $f$ by $\left(t^{-1} x_{1}, t x_{2}\right) \in \operatorname{SAut}\left(\mathbb{A}_{K\left[t^{ \pm 1}\right]}^{2}\right)$.

We can thus assume that $Q \neq 0$, denote by $d \geq 0$ the degree of $Q$ and by $\mu \in K^{*}$ the coefficient of degree $d$. We then choose some integer $a \geq 1$ such that $q=p^{a}>d$, write $\lambda=1 / \mu^{q} \in K^{*}$, and define

$$
\alpha=\left(\frac{x_{1}}{t^{d}}, t^{d} x_{2}+\lambda x_{1}^{q}+\frac{1}{t}\right) \in \operatorname{SAut}\left(\mathbb{A}_{K\left[t, t^{-1}\right]}^{2}\right) .
$$


A direct computation yields

$\alpha^{-1} f \alpha$

$=\left(x_{1}+t^{d} Q\left(t^{d} x_{2}+\lambda x_{1}^{q}+\frac{1}{t}\right), x_{2}+\frac{1}{t^{d}}+\frac{\lambda x_{1}^{q}}{t^{d}}-\frac{\lambda}{t^{d}}\left(x_{1}+t^{d} Q\left(t^{d} x_{2}+\lambda x_{1}^{q}+\frac{1}{t}\right)\right)^{q}\right)$.

The definition of $d$ and $\mu$ implies that we can write

$$
Q\left(t^{d} x_{2}+\lambda x_{1}^{q}+\frac{1}{t}\right)=\frac{\mu}{t^{d}}+\frac{P}{t^{d-1}},
$$

for some $P \in K\left[x_{1}, x_{2}, t\right]$. This yields (remembering that $\lambda \mu^{q}=1$ ), the following equality

$\alpha^{-1} f \alpha=\left(x_{1}+\mu+t P, x_{2}+\frac{1}{t^{d}}-\frac{\lambda}{t^{d}}(\mu+t P)^{q}\right)=\left(x_{1}+\mu+t P, x_{2}-\lambda t^{q-d} P^{q}\right)$.

Because $q>d$, the value of this element of $\operatorname{SAut}\left(\mathbb{A}_{K[t]}^{2}\right)$ at $t=0$ is $\left(x_{1}+\mu, x_{2}\right)$. Conjugating $\alpha^{-1} f \alpha$ with $\left(-\mu x_{2}, \mu^{-1} x_{1}\right)$ yields thus $F_{1}$.

To get $F_{2}$, we recall that $\left(t x_{1}, t^{-1} x_{2}\right)\left(x_{1}+\mu, x_{2}\right)\left(t^{-1} x_{1}, t x_{2}\right)=\left(x_{1}+t \mu, x_{2}\right)$. We are then tempted to use $\left(t x_{1}, t^{-1} x_{2}\right) \alpha^{-1} f \alpha\left(t^{-1} x_{1}, t x_{2}\right)$, but this element has in general no value at $t=0$. We then choose $m>0$ and define $\beta$ to be

$$
\beta=\left(\frac{x_{1}}{t^{m d}}, t^{m d} x_{2}+\lambda x_{1}^{q}+\frac{1}{t^{m}}\right) \in \operatorname{SAut}\left(\mathbb{A}_{K\left[t, t^{-1}\right]}^{2}\right) .
$$

Since $\beta$ is obtained from $\alpha$ by replacing $t$ with $t^{m}$, we obtain

$$
\beta^{-1} f \beta=\left(x_{1}+\mu+t^{m} P\left(x_{1}, x_{2}, t^{m}\right), x_{2}-\lambda t^{m(q-d)} P\left(x_{1}, x_{2}, t^{m}\right)^{q}\right) .
$$

We can now define $F_{2}(t)=\left(t x_{1}, t^{-1} x_{2}\right) \beta^{-1} f \beta\left(t^{-1} x_{1}, t x_{2}\right)$, which is equal to

$$
F_{2}(t)=\left(x_{1}+t \mu+t^{m+1} P\left(t^{-1} x_{1}, t x_{2}, t^{m}\right), x_{2}-\lambda t^{m(q-d)-1} P\left(t^{-1} x_{1}, t x_{2}, t^{m}\right)^{q}\right) .
$$

Choosing $m$ big enough, $F_{2}$ is defined at $t=0$ and $F_{2}(t)=\left(x_{1}, x_{2}\right)$.

4D. The diagonalisable elements. In order to finish the proof of Theorem 1.3 it remains to show that the conjugacy classes of diagonalisable elements is closed. This was shown in [Furter and Maubach 2010], for the group $\operatorname{Aut}\left(\mathbb{A}_{\mathbb{C}}^{2}\right)$, but with transcendental methods. In the case of $\operatorname{SAut}\left(\mathbb{A}_{\mathrm{k}}^{2}\right)$, we can however give a simple proof, that works for any algebraically closed field $\mathrm{k}$.

The proof uses the following result, which follows from the amalgamated structure product.

Lemma 4.11. Let $K$ be any field, let $\mu \in K^{*}, f=\left(\mu x_{1}, \mu^{-1} x_{2}\right) \in \operatorname{SAut}\left(\mathbb{A}_{K}^{2}\right)$, and $g \in \operatorname{SAut}\left(\mathbb{A}_{K}^{2}\right)$ be a conjugate of $f$ in this group. Then, there exists $\alpha \in \operatorname{SAut}\left(\mathbb{A}_{K}^{2}\right)$ such that

$$
g=\alpha^{-1} f \alpha \quad \text { and } \quad \operatorname{deg} \alpha \leq \operatorname{deg} g .
$$


Proof. We write $g=\alpha^{-1} f \alpha$ for some $\alpha \in \operatorname{SAut}\left(\mathbb{A}_{K}^{2}\right)$, and write $\alpha$ as a product of elements of $\operatorname{SAff}\left(\mathbb{A}_{K}^{2}\right)$ and $\operatorname{SJ}\left(\mathbb{A}_{K}^{2}\right)$, in a reduced way (no two consecutive elements belong to the same group). Note that $f$ is conjugate to $f^{-1}$ by $\left(x_{2},-x_{1}\right)$, so we can easily exchange $f$ with $f^{-1}$ if needed.

If $\alpha$ starts with an element $a \in \operatorname{SAff}\left(\mathbb{A}_{K}^{2}\right)$, then $a^{\prime}=a^{-1} j a \in \operatorname{SAff}\left(\mathbb{A}_{K}^{2}\right)$. If $a^{\prime}$ does not belong to $\operatorname{SJ}\left(\mathbb{A}_{K}^{2}\right)$, then $\operatorname{deg}(g)=\operatorname{deg}(\alpha) \operatorname{deg}\left(\alpha^{-1}\right)=\operatorname{deg}(\alpha)^{2}$ (the degree is the product of the Jonquières elements that occur, see Remark 4.2). If $a^{\prime}$ belongs to $\operatorname{SJ}\left(\mathbb{A}_{K}^{2}\right)$, then it is conjugate to $f$ or $f^{-1}$ in $\operatorname{SJ}\left(\mathbb{A}_{K}^{2}\right)$ (Proposition 4.9), and we can thus replace $a$ with an element of $\operatorname{SJ}\left(\mathbb{A}_{K}^{2}\right)$ and either decrease the length of $\alpha$ or it reduces to the case $\alpha \in \operatorname{SJ}\left(\mathbb{A}_{K}^{2}\right)$.

Suppose now that $\alpha$ starts with an element $j \in \operatorname{SJ}\left(\mathbb{A}_{K}^{2}\right)$. Replacing $j$ with $\rho j$, where $\rho$ is diagonal, we can assume that $j=\left(x_{1}+P\left(x_{2}\right), x_{2}+c\right)$, for some $P \in K\left[x_{2}\right]$ and $c \in K$, we thus obtain

$$
j^{\prime}=j^{-1} f j=\left(\mu x_{1}+P\left(x_{2}\right) \mu-P\left(x_{2}+c\left(\mu^{-1}-1\right)\right), \mu^{-1} x_{2}+c\left(\mu^{-1}-1\right)\right) \in \operatorname{SJ}\left(\mathbb{A}_{K}^{2}\right) .
$$

We have therefore $\operatorname{deg}\left(P\left(x_{2}\right)\right) \geq P\left(x_{2}\right) \mu-P\left(x_{2}+c\left(\mu^{-1}-1\right)\right)$, and if equality does not hold we can kill the highest coefficient of $P$ without changing $j^{\prime}=j^{-1} f j$. We reduce then to the case where $\operatorname{deg} j^{\prime}=\operatorname{deg} j$. If this degree is 1 , then $j \in \operatorname{SAff}\left(\mathbb{A}_{K}^{2}\right)$ and we can reduce the length of $\alpha$, or obtain $\operatorname{deg}(\alpha)=1$. The remaining case is when $\operatorname{deg} j^{\prime}=\operatorname{deg} j$. The result is then obtained if $\alpha=j$. Otherwise, $\alpha=j a_{1} j_{1} \ldots$ is a reduced writing, as well as $g=\cdots j_{1}^{-1} a_{1}^{-1} j^{\prime} a_{1} j_{1} \cdots$. We again find $\operatorname{deg} g \geq \operatorname{deg} \alpha$.

Proposition 4.12. Let $\mu \in \mathrm{k}^{*}, f=\left(\mu x_{1}, \mu^{-1} x_{2}\right) \in \operatorname{SAut}\left(\mathbb{A}_{\mathrm{k}}^{2}\right)$ and $d \geq 1$. The set

$$
C(f)=\left\{g f g^{-1} \mid g \in \operatorname{Aut}\left(\mathbb{A}_{\mathrm{k}}^{2}\right)\right\}=\left\{g f g^{-1} \mid g \in \operatorname{SAut}\left(\mathbb{A}_{\mathrm{k}}^{2}\right)\right\}
$$

is closed in $\operatorname{SAut}\left(\mathbb{A}_{\mathrm{k}}^{2}\right)$.

Proof. The equality between the two sets is obvious: if $g \in \operatorname{Aut}\left(\mathbb{A}_{\mathrm{k}}^{2}\right)$, we have $g^{\prime}=g \tau \in \operatorname{SAut}\left(\mathbb{A}_{\mathrm{k}}^{2}\right)$ for some diagonal $\tau \in \operatorname{Aut}\left(\mathbb{A}_{\mathrm{k}}^{2}\right)$, so $g f g^{-1}=g^{\prime} f g^{\prime-1}$. We can moreover assume that $\mu \neq 1$, otherwise the result is trivial. We fix some integer $d \geq 1$, write $Z=\operatorname{SAut}\left(\mathbb{A}_{\mathrm{k}}^{2}\right)_{\leq d}$ and we will show that $C(f) \cap Z$ is closed in $Z$ (this gives the result by Lemma 2.7).

We consider the morphism of algebraic varieties $Z \rightarrow \operatorname{Aut}\left(\mathbb{A}_{\mathrm{k}}^{2}\right)_{\leq d^{2}}$ given by $g \mapsto g f g^{-1}$ and denote by $Y \subset \operatorname{SAut}\left(\mathbb{A}_{\mathrm{k}}^{2}\right)_{\leq d}$ the preimage of $Z$. This gives rise to a morphism $\varphi: Y \rightarrow Z$, whose image is equal to $C(f) \cap Z$ by Lemma 4.11. By Corollary 2.26, it suffices to take an irreducible k-curve $\Gamma$, a rational map $\iota: \Gamma \rightarrow Y$ such that $\hat{\varphi}=\varphi \circ \iota: \Gamma \rightarrow Z$ is defined at a smooth point $p \in \Gamma$, and to show that the image $g=\hat{\varphi}(p) \in Z$ belongs to $C(f)$.

Note that $\iota$ corresponds to an element $\alpha \in \operatorname{Aut}\left(\mathbb{A}_{\mathrm{k}(\Gamma)}^{2}\right)$, and that $\hat{\varphi}$ corresponds to the element $\alpha f \alpha^{-1} \in \operatorname{Aut}\left(\mathbb{A}_{\mathrm{k}(\Gamma)}^{2}\right)$, which is defined at $p$ and whose value at $p$ 
corresponds to $g$. Since the set of algebraic elements is closed (Corollary 4.4), the element $g$ is conjugate to an element of $\operatorname{SJ}\left(\mathbb{A}_{\mathrm{k}}^{2}\right)$ (Remark 4.3). Looking at the actions on $\mathrm{k}(\Gamma)\left[x_{1}, x_{2}\right]$, we find that $f^{*}\left(x_{1}\right)=\mu x_{1}$ so $\left(\alpha f \alpha^{-1}\right)^{*}(v)=\mu v$, where $v=\left(\alpha^{-1}\right)^{*}\left(x_{1}\right) \in \mathrm{k}(\Gamma)\left[x_{1}, x_{2}\right]$. Replacing $v$ with $\lambda v$, where $\lambda \in \mathrm{k}(\Gamma)$, does not change the equation $\left(\alpha f \alpha^{-1}\right)^{*}(v)=\mu v$. We can therefore assume that $\alpha \in \mathcal{O}_{p}(\Gamma)\left[x_{1}, x_{2}\right]$ and that its value at $p$ is not zero. This shows that $\mu$ is also an eigenvalue of $g^{*}$. By Lemma 4.5, $g$ is conjugate in $\operatorname{SJ}\left(\mathbb{A}_{\mathrm{k}}^{2}\right)$ to $\left(v x_{1}, v^{-1} x_{2}\right)$, for some $v \in \mathrm{k} \backslash\{0,1\}$, or to $\left(\zeta x_{1}+x_{2}^{m-1} P\left(x_{2}^{m}\right), \zeta^{-1} x_{2}\right)$ for some primitive $m$-th root of unity $\zeta$, and $P \in \mathrm{k}\left[x_{2}\right] \backslash\{0\}$, and $\mu$ belongs to the subgroup of $\left(\mathrm{k}^{*}, \cdot\right)$ generated by $v$ or by $\zeta$, respectively. Let us observe that the second case is not possible. Indeed, otherwise we would have $f^{m}=\mathrm{id}$, so $\left(\alpha f \alpha^{-1}\right)^{m}=\mathrm{id}$ and thus $g^{m}=\mathrm{id}$, which is not the case since $P \neq 0$. We can thus assume, after composing $\alpha$ with an element of $\operatorname{SAut}\left(\mathbb{A}_{\mathrm{k}}^{2}\right)$, that $g=\left(v x_{1}, v^{-1} x_{2}\right)$ for some $v \in \mathrm{k} \backslash\{0,1\}$.

Denote by $U \subset \Gamma$ the dense open subset where $\iota$ is defined and where $\Gamma$ is smooth. Replacing $\Gamma$ with $U \cup\{p\}$, we can assume that $U=\Gamma \backslash\{p\}$ (if $p \in U$, the result is obvious), and thus that $\hat{\varphi}$ is a morphism $\hat{\varphi}: \Gamma \rightarrow Z$. Denote by $\kappa: \Gamma \rightarrow \mathbb{A}_{\mathrm{k}}^{2}$ the rational map given by $\kappa(u)=\iota(u)(0,0)$, when $u \in U$ (the image of the origin of $\mathbb{A}_{\mathrm{k}}^{2}$ ).

We now prove that $\kappa$ is defined at $p$. To do this, we define $F \subset \Gamma \times \mathbb{A}_{\mathrm{k}}^{2}$ to be the closed set $F=\left\{\left(c,\left(x_{1}, x_{2}\right)\right) \mid \hat{\varphi}(c)\left(x_{1}, x_{2}\right)=\left(x_{1}, x_{2}\right)\right\}$. Since $F$ is given by only two equations, each irreducible component of $F$ has dimension at least 1 . The intersection of $F$ with $u \times \mathbb{A}_{\mathrm{k}}^{2}$ yields one point, for each $u \in \Gamma$, hence the projection to $\Gamma$ yields an isomorphism $\pi: F \rightarrow \Gamma$ (because $\Gamma$ is smooth). The rational map $\Gamma \rightarrow F$ given by $u \mapsto(u, \kappa(u))$ corresponds to the identity, and is thus defined at $p$.

The map $\left(x_{1}, x_{2}\right) \mapsto\left(x_{1}, x_{2}\right)+\kappa(u)$ corresponds therefore to an element of $\operatorname{Aut}\left(\mathbb{A}_{\mathcal{O}(\Gamma)}^{2}\right)$, and replacing $\alpha$ with its composition by this one, we can assume that $\alpha$ fixes the origin. We obtain thus that the derivative of $\alpha f \alpha^{-1}$ at the origin is conjugate to $f$ by an element of $\operatorname{SL}(2, \mathrm{k})$, for each $u \in U$, so its eigenvalues are $\mu$ and $\mu^{-1}$, for any point of $U$. At the point $p$, we obtain $g=\left(v x_{1}, v^{-1} x_{2}\right)$, which implies that $v=\mu^{ \pm 1}$, so that $g$ and $f$ are conjugate in $\operatorname{SAut}\left(\mathbb{A}_{\mathrm{k}}^{2}\right)$ as we wanted.

Proof of Theorem 1.3. By Lemma 4.1 and Remarks 4.2 and 4.3, an algebraic element of $\operatorname{SAut}\left(\mathbb{A}_{\mathrm{k}}^{2}\right)$ is conjugate to an element of $\operatorname{SJ}\left(\mathbb{A}_{\mathrm{k}}^{2}\right)$ and a nonalgebraic element is conjugate to a Hénon automorphism, which is dynamically regular. In the latter case, the conjugacy class is closed by Theorem 1.1; this gives (3).

By Proposition 4.12, if $f \in \operatorname{SAut}\left(\mathbb{A}_{\mathrm{k}}^{2}\right)$ is diagonalisable, its conjugacy class is closed.

If $f \in \operatorname{SAut}\left(\mathbb{A}_{\mathrm{k}}^{2}\right)$ is algebraic but not diagonalisable, it is conjugate to an element of the families (ii), (iii) or (iv) of Lemma 4.5. The existence of the degeneration 
stated in (2) is given in Section 4C1 for families (ii), (iii) and in Proposition 4.10 for family (iv).

\section{Acknowledgements}

The author thanks Jean-Philippe Furter and Hanspeter Kraft for interesting discussions during the preparation of this article and for giving him access to the preprint [Furter and Kraft $\geq 2016$ ]. Thanks also to the referee for his precious remarks and corrections which improved the article.

\section{References}

[Bass et al. 1982] H. Bass, E. H. Connell, and D. Wright, "The Jacobian conjecture: reduction of degree and formal expansion of the inverse", Bull. Amer. Math. Soc. (N.S.) 7:2 (1982), 287-330. MR 663785 Zbl 0539.13012

[Bisi 2008] C. Bisi, "On commuting polynomial automorphisms of $\mathbb{C}^{k}, k \geq 3$ ", Math. Z. 258:4 (2008), 875-891. MR 2369061 Zbl 1161.32006

[Blanc 2010] J. Blanc, "Groupes de Cremona, connexité et simplicité”, Ann. Sci. Éc. Norm. Supér. (4) 43:2 (2010), 357-364. MR 2662668 Zbl 1193.14017

[Blanc and Cantat 2016] J. Blanc and S. Cantat, "Dynamical degrees of birational transformations of projective surfaces”, J. Amer. Math. Soc. 29:2 (2016), 415-471. MR 3454379

[Blanc and Furter 2013] J. Blanc and J.-P. Furter, “Topologies and structures of the Cremona groups", Ann. of Math. (2) 178:3 (2013), 1173-1198. MR 3092478 Zbl 1298.14020

[Demazure 1970] M. Demazure, "Sous-groupes algébriques de rang maximum du groupe de Cremona”, Ann. Sci. École Norm. Sup. (4) 3 (1970), 507-588. MR 0284446 Zbl 0223.14009

[Furter 1999] J.-P. Furter, "On the degree of iterates of automorphisms of the affine plane", Мапиscripta Math. 98:2 (1999), 183-193. MR 1667603 Zbl 0944.32017

[Furter 2009] J.-P. Furter, "Plane polynomial automorphisms of fixed multidegree", Math. Ann. 343:4 (2009), 901-920. MR 2471605 Zbl 1187.14064

[Furter and Kraft $\geq 2016$ ] J.-P. Furter and H. Kraft, "On the geometry of the automorphism group of affine $n$-space", in preparation.

[Furter and Lamy 2010] J.-P. Furter and S. Lamy, "Normal subgroup generated by a plane polynomial automorphism”, Transform. Groups 15:3 (2010), 577-610. MR 2718938 Zbl 1226.14080

[Furter and Maubach 2010] J.-P. Furter and S. Maubach, "A characterization of semisimple plane polynomial automorphisms", J. Pure Appl. Algebra 214:5 (2010), 574-583. MR 2577663 Zbl 1196.14056

[Guedj and Sibony 2002] V. Guedj and N. Sibony, "Dynamics of polynomial automorphisms of $\mathbb{C}^{k}$ ", Ark. Mat. 40:2 (2002), 207-243. MR 1948064 Zbl 1034.37025

[Jung 1942] H. W. E. Jung, "Über ganze birationale Transformationen der Ebene”, J. Reine Angew. Math. 184 (1942), 161-174. MR 0008915 Zbl 0027.08503

[van der Kulk 1953] W. van der Kulk, "On polynomial rings in two variables", Nieuw Arch. Wiskunde

(3) 1 (1953), 33-41. MR 0054574 Zbl 0050.26002

[Mumford 1970] D. Mumford, Abelian varieties, Tata Institute of Fundamental Research Studies in Mathematics 5, Oxford University Press, 1970. MR 0282985 Zbl 0223.14022

[Serre 2010] J.-P. Serre, "Le groupe de Cremona et ses sous-groupes finis", pp. 75-100 in Séminaire Bourbaki 2008/2009 (Exposé 1000), Astérisque 332, 2010. MR 2648675 Zbl 1257.14012 
[Shafarevich 1966] I. R. Shafarevich, "On some infinite-dimensional groups", Rend. Mat. e Appl. (5) 25:1-2 (1966), 208-212. MR 0485898 Zbl 0149.39003

[Sibony 1999] N. Sibony, "Dynamique des applications rationnelles de $\mathbb{P}^{k}$ ", pp. ix-x, xi-xii, 97-185 in Dynamique et géométrie complexes (Lyon, 1997), Panor. Synthèses 8, Soc. Math. France, Paris, 1999. MR 1760844 Zbl 1020.37026

Communicated by Hubert Flenner

Received 2015-01-02 Revised 2015-11-04 Accepted 2016-03-04

jeremy.blanc@unibas.ch Mathematisches Institut, Universität Basel, Spiegelgasse 1, CH-4051 Basel, Switzerland 


\section{Algebra \& Number Theory}

msp.org/ant

\section{EDITORS}

MANAGING EDITOR

Bjorn Poonen

Massachusetts Institute of Technology

Cambridge, USA

\author{
EDITORIAL BOARD CHAIR \\ David Eisenbud \\ University of California \\ Berkeley, USA
}

BOARD OF EDITORS

$\begin{aligned} \text { Dave Benson } & \text { University of Aberdeen, Scotland } & \text { Susan Montgomery } & \text { University of Southern California, USA } \\ \text { Richard E. Borcherds } & \text { University of California, Berkeley, USA } & \text { Shigefumi Mori } & \text { RIMS, Kyoto University, Japan } \\ \text { John H. Coates } & \text { University of Cambridge, UK } & \text { Raman Parimala } & \text { Emory University, USA } \\ \text { J-L. Colliot-Thélène } & \text { CNRS, Université Paris-Sud, France } & \text { Jonathan Pila } & \text { University of Oxford, UK } \\ \text { Brian D. Conrad } & \text { Stanford University, USA } & \text { Anand Pillay } & \text { University of Notre Dame, USA } \\ \text { Hélène Esnault } & \text { Freie Universität Berlin, Germany } & \text { Victor Reiner } & \text { University of Minnesota, USA } \\ \text { Hubert Flenner } & \text { Ruhr-Universität, Germany } & \text { Peter Sarnak } & \text { Princeton University, USA } \\ \text { Sergey Fomin } & \text { University of Michigan, USA } & \text { Joseph H. Silverman } & \text { Brown University, USA } \\ \text { Edward Frenkel } & \text { University of California, Berkeley, USA } & \text { Michael Singer } & \text { North Carolina State University, USA } \\ \text { Andrew Granville } & \text { Université de Montréal, Canada } & \text { Vasudevan Srinivas } & \text { Tata Inst. of Fund. Research, India } \\ \text { Joseph Gubeladze } & \text { San Francisco State University, USA } & \text { J. Toby Stafford } & \text { University of Michigan, USA } \\ \text { Roger Heath-Brown } & \text { Oxford University, UK } & \text { Ravi Vakil } & \text { Stanford University, USA } \\ \text { Craig Huneke } & \text { University of Virginia, USA } & \text { Michel van den Bergh } & \text { Hasselt University, Belgium } \\ \text { Kiran S. Kedlaya } & \text { Univ. of California, San Diego, USA } & \text { Marie-France Vignéras } & \text { Université Paris VII, France } \\ \text { János Kollár } & \text { Princeton University, USA } & \text { Kei-Ichi Watanabe } & \text { Nihon University, Japan } \\ \text { Yuri Manin } & \text { Northwestern University, USA } & \text { Efim Zelmanov } & \text { University of California, San Diego, USA } \\ \text { Philippe Michel } & \text { École Polytechnique Fédérale de Lausanne } & \text { Shou-Wu Zhang } & \text { Princeton University, USA }\end{aligned}$

PRODUCTION

production@msp.org

Silvio Levy, Scientific Editor

See inside back cover or msp.org/ant for submission instructions.

The subscription price for 2016 is US $\$ 290$ /year for the electronic version, and $\$ 485 /$ year (+\$55, if shipping outside the US) for print and electronic. Subscriptions, requests for back issues and changes of subscribers address should be sent to MSP.

Algebra \& Number Theory (ISSN 1944-7833 electronic, 1937-0652 printed) at Mathematical Sciences Publishers, 798 Evans Hall \#3840, c/o University of California, Berkeley, CA 94720-3840 is published continuously online. Periodical rate postage paid at Berkeley, CA 94704, and additional mailing offices.

ANT peer review and production are managed by EditFLow ${ }^{\circledR}$ from MSP.

\section{PUBLISHED BY}

- mathematical sciences publishers

nonprofit scientific publishing

http://msp.org/

() 2016 Mathematical Sciences Publishers 


\section{Algebra \& Number Theory}

Volume $10 \quad$ No. $5 \quad 2016$

Conjugacy classes of special automorphisms of the affine spaces

JÉRÉMY BLANC

Inversion of adjunction for rational and Du Bois pairs

SÁNDOR J. KOVÁCS and KARL SCHWEDE

Hochschild cohomology commutes with adic completion

LIRAN SHAUL

Bifurcations, intersections, and heights

LAURA DEMARCO

RANKEyA DATTA and KaREN E. SMITH

1091

Hoffmann's conjecture for totally singular forms of prime degree

STEPHEN SCULLY 